\title{
Liquid Biopsy-Based Colorectal Cancer Screening via Surface Markers of Circulating Tumor Cells
}

\author{
Francis Yew Fu Tieng ${ }^{1}{ }^{(D}$, Nadiah Abu ${ }^{1}$, Siti Nurmi Nasir ${ }^{1}$, Learn-Han Lee ${ }^{2, *(1)}$ \\ and Nurul-Syakima Ab Mutalib 1,2,3,*(D) \\ 1 UKM Medical Molecular Biology Institute (UMBI), Universiti Kebangsaan Malaysia, \\ Kuala Lumpur 56000, Malaysia; francistieng@yahoo.com.my (F.Y.F.T.); \\ nadiah.abu@ppukm.ukm.edu.my (N.A.); ctnurminasir@ppukm.ukm.edu.my (S.N.N.) \\ 2 Novel Bacteria and Drug Discovery Research Group, Microbiome and Bioresource Research Strength, \\ Jeffrey Cheah School of Medicine and Health Sciences, Monash University of Malaysia, \\ Subang Jaya 47500, Selangor, Malaysia \\ 3 Faculty of Health Sciences, Universiti Kebangsaan Malaysia, Kuala Lumpur 50300, Malaysia \\ * Correspondence: lee.learn.han@monash.edu (L.-H.L.); syakima@ppukm.ukm.edu.my (N.-S.A.M.); \\ Tel.: +60-391459073 (N.-S.A.M.)
}

Citation: Tieng, F.Y.F.; Abu, N.; Nasir, S.N.; Lee, L.-H.; Ab Mutalib, N.-S. Liquid Biopsy-Based Colorectal Cancer Screening via Surface Markers of Circulating Tumor Cells. Diagnostics 2021, 11, 2136. https:/ / doi.org/10.3390/diagnostics11112136

Academic Editors:

Lucía Inglada-Pérez and José Perea

Received: 30 September 2021

Accepted: 15 November 2021

Published: 17 November 2021

Publisher's Note: MDPI stays neutral with regard to jurisdictional claims in published maps and institutional affiliations.

Copyright: (c) 2021 by the authors. Licensee MDPI, Basel, Switzerland. This article is an open access article distributed under the terms and conditions of the Creative Commons Attribution (CC BY) license (https:// creativecommons.org/licenses/by/ $4.0 /)$.

\begin{abstract}
Colorectal cancer (CRC) is ranked second for cancer-related deaths worldwide with approximately half of the patients being diagnosed at the late stages. The untimely detection of CRC results in advancement to the metastatic stage and nearly $90 \%$ of cancer-related deaths. The early detection of CRC is crucial to decrease its overall incidence and mortality rates. The recent introduction of circulating tumor cells (CTCs) has enabled a less invasive sampling method from liquid biopsies, besides revealing key information toward CRC metastasis. The current gold standard for CTC identification is the CellSearch®system (Veridex). This first-generation instrumentation relies on a single cell surface marker (CSM) to capture and count CTCs. Detection of CTCs allows the identification of patients at risk for metastasis, whereas CTC enumeration could improve risk assessment, monitoring of systemic therapy, and detection of therapy resistance in advanced metastatic CRC. In this review, we compared the pros and cons between single CSM-based CTC enrichment techniques and multi-marker-based systems. We also highlighted the challenges faced in the routine implementation of CSM-dependent CTC detection methods in CRC screening, prediction, prognosis, disease monitoring, and therapy selection toward precision medicine, as well as the dwelling on post-CTC analysis and characterization methods.
\end{abstract}

Keywords: circulating tumor cells enrichment; cell surface markers; colorectal cancer; blood-based screening; non-invasive; multiplex

\section{Introduction}

Colorectal cancer (CRC) is among the most common global health issues with a startling surge in incidence (10.2\%) and mortality $(9.2 \%)$ rates [1]. The time of diagnosis directly corresponds to the overall survival of CRC patients. Localized cancer lesions could be easily removed during its early stages (5 year survival rate $>90 \%$ ) [2,3]. However, when CRC patients advance into the late/metastatic stage, only half of them survive within five years $[4,5]$. There is no effective treatment for patients diagnosed with metastasis or stage IV nonresectable tumors, and palliative therapies are often given only to relieve, delay, or prevent symptoms [6].

Currently, the gold standard for CRC diagnosis includes both colonoscopy [7] and histopathological examinations, where the two of them complement each other [8]. The former is the most sensitive procedure for CRC diagnosis as it permits the visualization and removal/surgery of colorectal tumors and pre-cancerous lesions (adenomas and polyps) $[9,10]$. When coupled with the latter, analysis of the excised tumors is possible, classifying them into different tumor stages/groups based on their clinicopathological features 
for better treatment selections [11]. For instance, the American Joint Committee on Cancer (AJCC) staging manual classifies CRC patients into five different stages (stage 0 to IV) based on the presence of tumors $(\mathrm{T})$, the number of lymph node metastases $(\mathrm{N})$, and the presence of distant metastases (M) $[12,13]$. However, these procedures are compromised and limited among patients due to their invasive nature, high cost, unstandardized protocols, expertise and apparatus requirements, labor-intensiveness, the low number of advanced neoplasms at specific sites and nonrepeatability over time, besides difficulties in predicting the extent of metastasis, distant metastases, and cancer heterogeneity accurately [14-18]. In short, the primary cause of cancer mortality is metastasis. Therefore, there is a need for a simpler, less invasive detection method to provide clear scientific evidence and improve early detection among CRC patients $[19,20]$.

\section{Circulating Tumor Cells Shed Insights toward Liquid Biopsy-based CRC Screening}

Circulating tumor cells (CTCs) denote epithelial cancer cells found in the bloodstream [21-23]. Their detection differs drastically from person to person but is often observed in patients with metastatic CRC (mCRC) [24-27]. Lately, CTCs have been intensively discussed due to the noninvasive identification technique, which enables the extraction of adequate diagnostic and predictive information directly from blood as compared to the invasive conventional biopsy [28]. In addition, CTCs have the potential to provide direct access to all phases of CRC carcinogenesis and are the main vehicles for metastatic reoccurrence [29,30]. During metastasis, tumor cells (1 million cells per gram of tumor tissue) escape from the tumor mass into the bloodstream, forming CTCs [22,31]. Although the majority of the CTCs undergo cell detachment-induced apoptosis, a small portion of the cells survive and form a micrometastasis [32-34]. From there, only a few of them progress to form macroscopic tumors [35,36]. These cells aggregate and form secondary tumor sites, which then release CTCs back into the bloodstream and might attach to the initial primary site (local relapse), as illustrated in Figure 1 [37,38]. In short, CTCs are believed to generate valuable information and provide critical insights into the aggressive nature of the tumor and also a better understanding of the underlying biology related to CRC dissemination and metastasis [21,39]. Thus, the application of CTCs as cancer biomarkers offers a more effective alternative to detect, analyze, treat, and monitor CRC therapeutic responses and disease progression $[40,41]$.

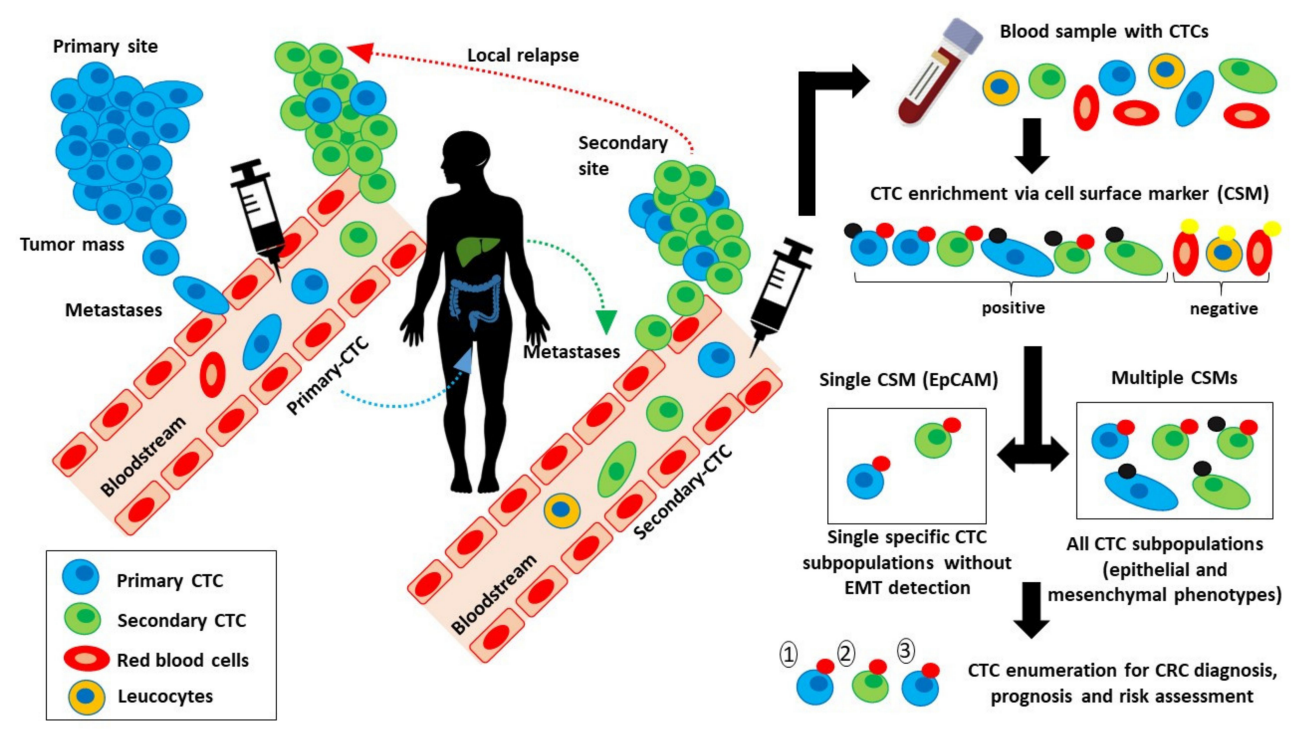

Figure 1. Sources of CTCs and the comparison between single-based and multi-marker-based CSM-dependent CTC enrichment. 


\section{Existing Blood-based Biomarkers Are Not Effective with Low Accuracy}

Previous studies had proven the implementation of biomarkers from blood circulation as a noninvasive method for CRC screening, particularly during its early stages (stage I or premalignant stage) [42-49]. Despite the discovery of innumerable blood-based CRCspecific markers, follow-up cohort studies including a large population of patients are lacking, and relatively few of them could be translated into clinical practice [50-54].

To date, the two most recognized CRC-specific antigens are carcinoembryonic antigen (CEA) and carbohydrate antigen (CA 19.9) [55-59]. However, both of these biomarkers are not effective in CRC detection, due to the overlapping/close proximity of ranges of concentration across different stages [60] and the fact that only certain CRC patients have expressed elevations in CEA (43\%) and CA19.9 (27\%) in blood serum, hindering accurate distinction [61]. Other factors include the nonspecificity of CEA and CA 19.9 toward a particular histological type or origin of the carcinoma, false-positive results from the elevation of CEA levels due to benign conditions (e.g. hepatitis, pancreatitis, obstructive pulmonary disease, and inflammatory bowel disease), and analytical variables such as variations in sampling and storage methods, patients' condition, and stability of the biomarkers $[62,63]$. Thus, the identification of a rapid, sensitive, and CRC marker-specific method is crucial in developing accurate assays for effective CRC detection from peripheral blood [64].

\section{4. "Gold Standard": Single CTC-Specific Cell Surface Marker-Positive Enrichment}

The pioneer stage in applying CTCs as cancer biomarkers is the ability to capture and detect CTCs from blood samples [65]. The detection of CTCs is challenging majorly because of its rarity (1 CTC per $10^{7}$ to $10^{9}$ hematological cells $/ \mathrm{mL}$ ), the short half-life of a few hours ex vivo, the lack of a single ubiquitous/universal CTC-specific marker, and technical limitations such as low separation efficiency and low recovery rates [66-71]. This has led to the invention of isolation devices that focus on exploiting cell surface markers (CSMs)/antigens expressed on CTCs but not expressed on the surrounding nontarget cells (e.g., leucocytes and red blood cells). Some examples of the CTC enrichment techniques are immunoaffinity-based purification (biological), and biophysical isolation methods that rely on the differential size and/or density of CTCs and di-electrophoretic-based strategies [72,73]. Among them, CTC enrichment by immunoaffinity is the most widely used strategy for CTC isolation.

Immunoaffinity-based CTC purification is categorized into two main groups, namely positive and negative enrichment strategies. Positive enrichment isolates CTCs by targeting specific CSMs, whereas the latter captures background/nontarget cells by targeting CSMs deficient in CTCs. Currently, the most well-known and established positive enrichment method is the CellSearch ${ }^{\circledR}$ system (Veridex). This Food and Drug Administration (FDA)approved first-generation instrumentation relied on a single CSM epithelial cell adhesion molecule (EpCAM) to capture CTCs, followed by CTC enumeration to provide cancer prediction, prognosis, and clinical outcomes [74-78]. The detection of CTCs enabled the identification of patients at risk for metastasis originating from localized CRC, whereas CTC enumeration could improve risk assessment, monitoring of systemic therapy, and detection of therapy resistance in advanced mCRC. Since its approval, enrichment techniques based on a single specific CSM have become the gold standard for CTC isolation [79,80]. To summarize, the primary principle of this method includes both targeting the antigen expression of CTCs (detection) and counting of CTC (enumeration).

Despite the advancement in monoclonal antibodies, microfluidics, fluorescence, and laser technologies, EpCAM remains the principal CSM for most of the CTCs enrichment methods available for CRC [81]. In 2019, Gupta and the coworkers evaluated the assay specificity and clinical feasibility of the CellMax CTC detection assay (CellMax Life) in a cohort study. This single specific EpCAM-dependent assay that is based on microfluidic chip technology could accurately enrich CTC from peripheral blood with a high sensitivity $(80 \%)$, specificity $(80 \%)$, and recovery rate up to $80.8 \%$ when spiked with HT29 cells [82]. 
In the same year, Tsai et al. verified the single EpCAM-dependent CellMax platform as an early cancer detection method due to its ability to relate the captured epithelial CTCs count to different CRC stages (adenomas, stage I, II, III, IV) with a positive detection rate up to $94.5 \%$ (307/325 patients) [30]. Following this, a polymeric chip coated with solely EpCAM was developed by Kure and the coauthors to enrich CTCs from CRC patients. This protocol not only showed a significantly higher positive detection rate than the CA19.9 test but also validated CTCs as effective markers for stage II and III CRC, who often exhibit negative conventional serum marker test results [83]. In 2020, a group of Australian researchers applied the single EpCAM-based magnetic CTC isolation technique known as IsoFlux (Fluxion Biosciences) in a comparative, longitudinal study. Interestingly, they discovered that CTCs with high microsatellite instability were associated with a rise in CTCs released intra-operatively and post-operatively [84]. In a nutshell, the single specific CSM-based CTC enrichment technique is capable of CRC early screening, prognosis, and prediction of treatment, as well as disease progression/treatment effects monitoring.

\section{Single Specific CSM-based CTC Enrichment Strategy Had Its Limitations}

Although targeting EpCAM on CTCs from CRC (epithelial origin) seems to be the best option to distinguish between CTCs and normal blood cells with mesenchymal phenotypes, the overreliance on a single specific CSM resulted in a selection bias [79], where only CTCs that predominantly retain epithelial characteristics (high EpCAM levels) are enriched, excluding a subpopulation of CTCs with mesenchymal traits (low or no EpCAM expressed) [85-87]. This could have serious implications as CTCs are characterized by phenotypic plasticity that mainly reflects an epithelial-to-mesenchymal transition state (EMT), especially when progressing into mCRC and/or acquiring chemoresistance [88-94]. For instance, scientists from China had found out that only mesenchymal and epithelialmesenchymal CTCs, not epithelial CTCs, were correlated with clinical stage and metastasis in CRC [95]. Moreover, increased analytic sensitivity and specificity by including more CSM markers for secondary CTC identification after the initial single EpCAM-positive enrichment did not change the fact that only EpCAM-positive CTCs were isolated. Thus, it was unsurprising that a prospective and investigator-blinded side-by-side comparison of CellSearch (pan-CK) and GILUPI CellCollector (EpCAM and pan-CK double staining) did not show significance in either the total number or the frequency of CTCs detected in both metastatic and nonmetastatic CRC patients [96]. In this context, the heterogeneity of CTCs creates a significant loss of certain CTC subpopulations, which leads to uncertainty in the accuracy of a single CSM-dependent positive analysis to identify a patient's CTC status.

To overcome this, negative enrichment was introduced. It captures nontarget cells (e.g., hematogenous cells), followed by the isolation of CTCs. Unlike positive enrichments, negative enrichments could harvest all types of CTCs as they are not dependent on the CSM profiles and are more competent for the discovery of cellular and transcriptomic cancer biomarkers of cancer and downstream analyses such as genetic assays, CTC culture, and xenografts [97]. Regardless of several negative methods developed such as the CellSearch ${ }^{\circledR}$ system (Veridex) [98], Cyttel method [99], RosetteSep ${ }^{\mathrm{TM}}$ system [100], subtraction enrichment, and immunostaining-fluorescence in situ hybridization (SE-iFISH) (Cytelligen) [101] and EasySep ${ }^{\mathrm{TM}}$ (StemCell Technologies) [102], all of them use lymphocyte common antigen (CD45) as the main marker to remove hematogenous cells. Similar to traditional negative enrichment, these systems employ the single specific CD45 CSM to deplete nontarget cells and elute CTCs, followed by a CTC-specific antibody cocktail (EpCAM, CK, CK3, CK18, CK19, MUC1, CD44, CD133, ALDH1, and/or CEP8) to identify the captured cells. The downside of the single CSM negative isolation is that it had less purity (ability to detect CTCs in the presence of contaminating background cells) and lower specificity (significant loss of CTCs) than positive enrichment. 


\section{Alternative CSMs and Multiplexing Show Potential in Targeting a Wider CTC Population}

To further expand the detection limit of single analyte-dependent enrichment (to include more CTC subpopulations during separation), several attempts had been conducted using different CSMs, including KRAS (Kirsten rat sarcoma viral oncogene), pancytokeratin (pan-CK), vimentin (VIM), cluster of differentiation (CD2, CD16, CD19, CD36, CD38, CD45, and CD66b), and/or glycophorin A. For instance, Feng and the researchers proposed the use of a lipid magnetic ball coated with KRAS to isolate CTCs from CRC with KRAS mutations. The reason for opting for KRAS over EpCAM was that (1) KRAS was closely related to CRC signaling pathways such as MAPK, PI3K, Wnt, and EGFR [103-106]; (2) almost half of the CRC patients were characterized by a mutation in the codons 12 and 13 in exon 2 of the KRAS gene [107,108]; and (3) those without the KRAS mutation tend to develop secondary KRAS mutations $(\sim 30 \%)$ during courses of targeted therapy $[109,110]$. Based on their results, KRAS-modified enrichment could effectively improve the capture ability of CTCs with KRAS mutation up to $92.9 \%$, and the result was in concordance with clinical diagnosis and pathology. Their results showed that KRAS immune lipid magnetic balls could be used in the diagnosis and treatment of KRAS CRC [111].

In tackling the challenges of single CSM-dependent CTC enrichment in addressing the totality of CTCs, a multi-marker-based system could potentially isolate CTCs of different origins by covering epithelial, mesenchymal, and stem cell markers. In 2017, Soler et al. modified the RosetteSep ${ }^{\mathrm{TM}}$ System (StemCell Technologies) to include a list of CSMs (CD2, CD16, CD19, CD36, CD38, CD45, CD66b, and glycophorin A) as tetrameric antibody complexes to crosslink unwanted cells for CTC elution. CTCs were then purified via density gradient centrifugation, followed by an EPISPOT assay where specific secreted proteins were captured by an antibody-coated membrane. These immunospots were counted (one immunospot corresponded to the protein fingerprint of one viable cell). Their experiment described negative enrichment multiplexing to be capable of harvesting all types of CTCs, detecting viable CTCs at the single-cell resolution and providing downstream analysis for CTC phenotypic and protein characterization [100].

Following this, a group of scientists from France isolated CTCs from healthy blood cells via CD45 depletion (RosetteSep ${ }^{\mathrm{TM}}$ system) as a pre-enrichment step, followed by CTC identification with three CSM markers: EpCAM, pan-CK, and VIM. EpCAM and pan-CK recognized antigens/epitopes present on epithelial CTCs, whereas VIM captured CTCs undergoing EMT. They invented a simple, fast, sensitive, and higher recovery technique to detect both epithelial and mesenchymal CTCs that could be complemented, when needed, by other in-depth analyses [112]. In 2020, Hamid et al. claimed that prior to the CD45-based subtraction of hematogenous cells, CTC enrichment with both EpCAM and CK markers enabled the authors to relate CRC staging with CTC morphological and phenotype features [102].

On the other hand, Bahnassy and the coresearchers combined multiple enrichment methods to identify CTCs. Briefly, CD45-based negative enrichment was utilized to subtract nontarget cells. The validation of CTC was performed by a combination of CellSearch, cytomorphology, flow cytometry (FCM), and real transcriptase quantitative PCR (RT-qPCR) with multiple markers including cytokeratin (CK3, CK19), mucin 1 (MUC1), CD44, CD133, and aldehyde dehydrogenase 1 (ALDH1). In this comparative study, they confirmed the superiority of multiplexing several different techniques (positive detection rate: $68.3 \%$ ) over a single CTC enrichment strategy (positive detection rate: 54\% (CellSearch); 50.8\% $(\mathrm{FCM})$ ). Interestingly, CTCs were identified as novel therapeutic targets for nonmetastatic CRC [98]. To sum up, despite numerous benefits over traditional single CSM-based CRC detection, multiplexing on CTC enrichment remains very limited [113].

\section{Circulating Cancer Stem Cells Are a Rare CTC Subtype}

Until today, the mechanisms between CTCs and circulating cancer stem cells (CCSCs) remain unclear [114]. There are, however, increasing evidence revealing the existence 
of cellular heterogeneity within CTCs [40], and that a presumably small subset of them harbor cancer stem cell characteristics due to their ability to survive in the blood, and resist chemotherapy and progression into metastatic lesions [115-117]. For instance, in 2017, Grillet et al. demonstrated that CTCs from CRC patients exhibited cancer stem cell hallmarks when culturing ex vivo [115]. Furthermore, CTCs and CCSCs show different functional states of the same pathogenically relevant cancer cell subpopulations $[118,119]$. Thus, the identification of drug-resistant CTCs in the bloodstream would, at least theoretically, provide a unifying hypothesis, where CCSCs might be a rare CTC subtype [120].

As CCSCs are likely to represent small subsets of CTCs, the traditional CSM-based CTC enrichment method could be applied in identifying CCSCs. In 2020, a group of researchers in Italy shed insights toward the possibility of using the anti-human CD44v6 antibody to detect the CCSC subpopulation from patients-derived CTCs. The CD44v6 isoform was selected for several reasons: (1) involvement in cancer cell migration and invasion; (2) functional biomarker of stemness and therapeutic target in CRC [121]; (3) presence in all CRC stem cells (capable of metastatic tumors generation) [122]; and (4) the highly expressed CD44v6 protein on CTCs with functional attributes of CCSCs [123]. Briefly, a single CSM EpCAM was selected to positively enrich CTCs from peripheral blood. The isolated CTCs were then verified with CK8, CK18, CK19, CD44v6, and CD45. Their research demonstrated that the enumeration of CD44v6-positive CTC/CCSC obtained from mCRC patients could be used to early detect intrinsic drug resistance, as well as predict the first-line treatment failure [124]. In short, the CSM-based enrichment technique showed potential in isolating CCSCs for CRC screening and tumor response prediction in mCRC patients. Table 1 summarizes the common CTC enrichment techniques categorized by specific CSMs for CRC screening. 
Table 1. CTC-specific cell surface marker (CSM)-based enrichment techniques for CRC screening.

\begin{tabular}{|c|c|c|c|c|c|c|c|c|c|}
\hline Feature & $\begin{array}{l}\text { Surface } \\
\text { Marker }\end{array}$ & $\begin{array}{c}\text { CTC } \\
\text { Identification } \\
\text { Marker }\end{array}$ & $\begin{array}{c}\text { CTC } \\
\text { Enrichment } \\
\text { Technique }\end{array}$ & Principle/Technology & Pros and Cons & $\begin{array}{c}\text { Positive } \\
\text { Detection Rate }\end{array}$ & Study & $\begin{array}{l}\text { Clinical } \\
\text { Utility }\end{array}$ & Ref \\
\hline \multirow{5}{*}{$\begin{array}{l}\text { Single } \\
\text { specific } \\
\text { CSM }\end{array}$} & EpCAM & $\begin{array}{c}\text { CK8, CK18, CK19, } \\
\text { HER2, CD45, } \\
\text { DAPI, Hoechst }\end{array}$ & $\begin{array}{l}\text { CellSearch®system } \\
\text { (Veridex) }\end{array}$ & $\begin{array}{l}\text { Positive enrichment; ferromagnetic } \\
\text { beads labeled with EpCAM-antibodies } \\
\text { to capture CTCs; identification of CTCs } \\
\text { via staining with CK8, CK18, CK19, } \\
\text { and HER2; CD } 45 \text { marker to exclude } \\
\text { hematogenous cells; DAPI/Hoechst as } \\
\text { marker to identify intact CTCs; CTC } \\
\text { enumeration via CellTrack Analysis II }\end{array}$ & $\begin{array}{l}\text { FDA-approved for } \\
\text { advanced CRC; loss of } \\
\text { EpCAM-negative CTCs; } \\
\text { lack of EMT detection; no } \\
\text { further downstream } \\
\text { analysis }\end{array}$ & $\begin{array}{c}88.9 \%(32 / 36 \\
\text { patients })\end{array}$ & $\begin{array}{l}\text { Prospective, } \\
\text { multicenter, } \\
\text { nonrandomized trial } \\
\text { (NCT00994864) }\end{array}$ & $\begin{array}{l}\text { Prognosis and } \\
\text { prediction of } \\
\text { CRC }\end{array}$ & {$[74]$} \\
\hline & EpCAM & $\begin{array}{l}\text { CK20, CD } 45, \\
\text { DAPI }\end{array}$ & $\begin{array}{l}\text { CellMax platform } \\
\text { (CellMax Life) }\end{array}$ & $\begin{array}{l}\text { Positive enrichment; microfluidics chip } \\
\text { technology platform; EpCAM-coated } \\
\text { SLB to capture CTCs; identification of } \\
\text { CTCs via staining with CK20; CTCs } \\
\text { enumeration via AI-based automated } \\
\text { CellReviewer }\end{array}$ & $\begin{array}{l}\text { Loss of EpCAM-negative } \\
\text { CTCs; lack of EMT } \\
\text { detection; CTCs intact for } \\
\text { downstream analysis }\end{array}$ & $\begin{array}{l}43.8 \%(14 / 32 \\
\text { patients })\end{array}$ & Cohort study & $\begin{array}{c}\text { CRC } \\
\text { screening }\end{array}$ & [82] \\
\hline & EpCAM & $\begin{array}{l}\text { CK20, CD45, } \\
\text { DAPI }\end{array}$ & $\begin{array}{l}\text { CellMax platform } \\
\text { (CellMax Life) }\end{array}$ & $\begin{array}{c}\text { Positive enrichment; CTCs enrichment } \\
\text { using EpCAM antibody and stained } \\
\text { using CK20 for confirmation; CTC } \\
\text { enumeration via an algorithm in } \\
\text { CellFinder software }\end{array}$ & $\begin{array}{l}\text { Loss of EpCAM-negative } \\
\text { CTCs; lack of EMT } \\
\text { detection }\end{array}$ & $\begin{array}{c}94.5 \%(307 / 325 \\
\text { patients })\end{array}$ & $\begin{array}{l}\text { Bioanalytical assay } \\
\text { development and } \\
\text { validation study }\end{array}$ & $\begin{array}{c}\text { CRC } \\
\text { screening }\end{array}$ & {$[30]$} \\
\hline & EpCAM & $\begin{array}{c}\text { CK8, CK18, CD45, } \\
\text { DAPI }\end{array}$ & $\begin{array}{l}\text { Polymeric CTC } \\
\text { chip }\end{array}$ & $\begin{array}{c}\text { Positive enrichment; polymeric } \\
\text { microfluidic chip coated with EpCAM } \\
\text { for CTC detection; staining with CK8 } \\
\text { and CK18 for CTC validation; manual } \\
\text { CTC enumeration under an inverted } \\
\text { fluorescence microscope }\end{array}$ & $\begin{array}{l}\text { Loss of EpCAM-negative } \\
\text { CTCs; easily blocked chips; } \\
\text { lack of EMT detection }\end{array}$ & $\begin{array}{c}92.3 \%(12 / 13 \\
\text { patients })\end{array}$ & $\begin{array}{c}\text { Comparative, } \\
\text { longitudinal study }\end{array}$ & $\begin{array}{l}\text { CRC } \\
\text { screening; } \\
\text { CRC } \\
\text { progression } \\
\text { monitoring } \\
\text { and treatment } \\
\text { effects }\end{array}$ & [83] \\
\hline & EpCAM & $\begin{array}{l}\text { CK-7, CK-8, } \\
\text { CK-18, CK-19, } \\
\text { CD45, DAPI, } \\
\text { Hoechst }\end{array}$ & $\begin{array}{l}\text { IsoFlux (Fluxion } \\
\text { Biosciences) }\end{array}$ & $\begin{array}{l}\text { Positive enrichment; EpCAM- based } \\
\text { magnetic separation by flow cytometry } \\
\text { to capture CTCs; identification of CTC } \\
\text { via CK-7, CK-8, CK-18, and CK-19 } \\
\text { markers; CTC enumeration via IsoFlux }\end{array}$ & $\begin{array}{l}\text { Loss of EpCAM-negative } \\
\text { CTCs; detection of CTC } \\
\text { and MSI status in the } \\
\text { peri-operative colorectal } \\
\text { surgery setting; lack of } \\
\text { EMT detection; no further } \\
\text { downstream analysis }\end{array}$ & $\begin{array}{l}95 \%(19 / 20 \\
\text { patients) }\end{array}$ & $\begin{array}{l}\text { Cross-sectional } \\
\text { study }\end{array}$ & $\begin{array}{l}\text { CRC } \\
\text { screening; } \\
\text { CRC } \\
\text { progression } \\
\text { monitoring } \\
\text { and treatment } \\
\text { effects }\end{array}$ & {$[84]$} \\
\hline
\end{tabular}


Table 1. Cont

\begin{tabular}{|c|c|c|c|c|c|c|c|c|c|}
\hline Feature & $\begin{array}{l}\text { Surface } \\
\text { Marker }\end{array}$ & $\begin{array}{c}\text { CTC } \\
\text { Identification } \\
\text { Marker }\end{array}$ & $\begin{array}{c}\text { CTC } \\
\text { Enrichment } \\
\text { Technique }\end{array}$ & Principle/Technology & Pros and Cons & $\begin{array}{c}\text { Positive } \\
\text { Detection Rate }\end{array}$ & Study & $\begin{array}{l}\text { Clinical } \\
\text { Utility }\end{array}$ & Ref. \\
\hline & $\begin{array}{l}\text { EpCAM or } \\
\text { KRAS }\end{array}$ & $\begin{array}{l}\text { CK20, CD } 45, \\
\text { DAPI }\end{array}$ & $\begin{array}{l}\text { MACS/ } \\
\text { microemulsion } \\
\text { method }\end{array}$ & $\begin{array}{c}\text { Positive enrichment; EpCAM or } \\
\text { KRAS-coated lipid bilayer } \\
\text { encapsulated superparamagnetic } \\
\mathrm{Fe}_{3} \mathrm{O}_{4} \text { nanoparticles balls to capture } \\
\mathrm{CTCs} \text { with EpCAM expression or } \\
\text { KRAS mutation; identification of CTC } \\
\text { via CK20 and CD45; KRAS detection } \\
\text { via PCR }\end{array}$ & $\begin{array}{l}\text { Detection of CTCs with } \\
\text { KRAS mutation; intact } \\
\text { CTCs for PCR to validate } \\
\text { KRAS mutation }\end{array}$ & $\begin{array}{c}100 \% \text { (KRAS) } \\
\text { (55/55 patients); } \\
54.5 \% \text { (EpCAM) } \\
\text { (30/55 patients) }\end{array}$ & Comparative study & $\begin{array}{l}\text { KRAS } \\
\text { mutation } \\
\text { detection; } \\
\text { provide } \\
\text { diagnosis and } \\
\text { treatment of } \\
\text { KRAS CRC }\end{array}$ & [111] \\
\hline & EpCAM & $\begin{array}{c}\text { pan-CK, CD45, } \\
\text { DAPI, Hoechst } \\
\text { EpCAM, pan-CK, } \\
\text { CD45, DAPI, } \\
\text { Hoechst }\end{array}$ & $\begin{array}{l}\text { CellSearch }{ }^{\circledR} \text { system } \\
\text { (Veridex) } \\
\text { GILUPI } \\
\text { CellCollector }\end{array}$ & $\begin{array}{c}\text { Positive enrichment; ferromagnetic } \\
\text { beads labeled with EpCAM antibodies; } \\
\text { identification of CTCs via staining with } \\
\text { pan-CK } \\
\text { Positive enrichment; novel in vivo CTC } \\
\text { detection device with EpCAM, } \\
\text { followed by pan-CK/EpCAM } \\
\text { (double-staining) for verification }\end{array}$ & $\begin{array}{l}\text { FDA-approved; loss of } \\
\text { EpCAM-negative CTCs; } \\
\text { lack of EMT detection } \\
\text { Loss of EpCAM-negative } \\
\text { CTCs; lack of EMT } \\
\text { detection }\end{array}$ & $\begin{array}{l}31.3 \%(25 / 80 \\
\text { patients }) \\
41.3 \%(33 / 80 \\
\text { patients })\end{array}$ & $\begin{array}{l}\text { Prospective, single } \\
\text { center, } \\
\text { investigator-blinded } \\
\text { side-by-side } \\
\text { comparative study }\end{array}$ & $\begin{array}{l}\text { Prediction of } \\
\text { CRC (overall } \\
\text { survival based } \\
\text { on staging) } \\
\text { CRC } \\
\text { screening }\end{array}$ & [96] \\
\hline & EpCAM & $\begin{array}{l}\text { CK8, CK18, CK19, } \\
\text { CD44v6, CD45 }\end{array}$ & $\begin{array}{l}\text { CellSearch }{ }^{\circledR C X C} \\
\text { kit (Menarini } \\
\text { Silicon } \\
\text { Biosystems) }\end{array}$ & $\begin{array}{l}\text { Positive enrichment; ferromagnetic } \\
\text { beads labeled with EpCAM-antibodies } \\
\text { to capture CTCs; identification of } \\
\text { CCSCs via CD44v6 expression }\end{array}$ & $\begin{array}{l}\text { Identification of CTCs } \\
\text { with functional attributes } \\
\text { of CCSCs via CD } 44 \mathrm{v} 6 \\
\text { expression }\end{array}$ & $\begin{array}{l}62.5 \%(25 / 40 \\
\text { patients })\end{array}$ & $\begin{array}{l}\text { Bioanalytical assay } \\
\text { development study }\end{array}$ & $\begin{array}{l}\text { mCRC } \\
\text { screening; } \\
\text { prediction of } \\
\text { first-line } \\
\text { treatment } \\
\text { failure and } \\
\text { tumor } \\
\text { response in } \\
\text { mCRC } \\
\text { patients }\end{array}$ & [124] \\
\hline & CD45 & DAPI & Cyttel method & $\begin{array}{c}\text { Negative enrichment; CD45-based } \\
\text { immunomagnetic system to remove } \\
\text { hematogenous cells; CTC identification } \\
\text { via imFISH of chromosomes } 8 \text { and } 17 \\
\text { H1 fluorescent probes, together with } \\
\text { DAPI staining }\end{array}$ & $\begin{array}{l}\text { Loss of significant cells; } \\
\text { low purity }\end{array}$ & $\begin{array}{l}58.7 \%(71 / 121 \\
\text { patients })\end{array}$ & Retrospective study & $\begin{array}{l}\text { CRC } \\
\text { screening; } \\
\text { prediction of } \\
\text { survival } \\
\text { outcome }\end{array}$ & [99] \\
\hline
\end{tabular}


Table 1. Cont.

\begin{tabular}{|c|c|c|c|c|c|c|c|c|c|}
\hline Feature & $\begin{array}{l}\text { Surface } \\
\text { Marker }\end{array}$ & $\begin{array}{c}\text { CTC } \\
\text { Identification } \\
\text { Marker }\end{array}$ & $\begin{array}{c}\text { CTC } \\
\text { Enrichment } \\
\text { Technique }\end{array}$ & Principle/Technology & Pros and Cons & $\begin{array}{c}\text { Positive } \\
\text { Detection Rate }\end{array}$ & Study & $\begin{array}{l}\text { Clinical } \\
\text { Utility }\end{array}$ & Ref. \\
\hline $\begin{array}{l}\text { Multi- } \\
\text { CSM }\end{array}$ & $\begin{array}{c}\text { CD2, CD16, } \\
\text { CD19, CD36, } \\
\text { CD38, CD45, } \\
\text { CD66b, and } \\
\text { glycophorin A }\end{array}$ & CK19, VEGF & $\begin{array}{c}\text { RosetteSep }^{\mathrm{TM}} \\
\text { System (StemCell } \\
\text { Technologies) }\end{array}$ & $\begin{array}{l}\text { Positive enrichment; immunodensity } \\
\text { procedure; RosetteSep }{ }^{\mathrm{TM}} \text { tetrameric } \\
\text { antibody complexes crosslink } \\
\text { unwanted hematogenous cells; } \\
\text { isolation of CTCs via density gradient } \\
\text { centrifugation, followed by EPISPOT } \\
\text { assay where specific secreted proteins } \\
\text { were captured by antibody-coated } \\
\text { membrane; counting of immunospots } \\
\text { (one immunospot corresponded to the } \\
\text { protein fingerprint of one viable cell) }\end{array}$ & $\begin{array}{l}\text { Many CTCs harvested; } \\
\text { detection of viable CTCs at } \\
\text { the single-cell resolution; } \\
\text { utilization of CTC-secreted } \\
\text { proteins for enrichment; } \\
\text { possible for protein } \\
\text { characterization }\end{array}$ & - & $\begin{array}{l}\text { Bioanalytical assay } \\
\text { development study }\end{array}$ & $\begin{array}{l}\text { CRC } \\
\text { screening; } \\
\text { possible } \\
\text { protein charac- } \\
\text { terization }\end{array}$ & [100] \\
\hline \multirow{3}{*}{$\begin{array}{l}\text { Post } \\
\text { multi- } \\
\text { CSM }\end{array}$} & $\mathrm{CD} 45$ & $\begin{array}{c}\text { pan-CK, EPCAM, } \\
\text { VIM, DAPI }\end{array}$ & $\begin{array}{c}\text { RosetteSep }^{\mathrm{TM}} \\
\text { System (StemCell } \\
\text { Technologies) }\end{array}$ & $\begin{array}{l}\text { Pre-negative CD45 enrichment to } \\
\text { exclude hematogenous cells; secondary } \\
\text { enrichment with EpCAM and CK. } \\
\text { Pan-CK, EPCAM, and VIM; CTC } \\
\text { enumeration via FCM }\end{array}$ & $\begin{array}{l}\text { Simple, fast, sensitive, and } \\
\text { higher recovery to detect } \\
\text { potential CTCs (with } \\
\text { EMT); CTCs intact for } \\
\text { downstream analysis }\end{array}$ & $\begin{array}{c}46.7 \%(7 / 15 \\
\text { patients) }\end{array}$ & Case control & $\begin{array}{c}\text { CRC } \\
\text { screening }\end{array}$ & [112] \\
\hline & $\mathrm{CD} 45$ & EpCAM, CK & $\begin{array}{c}\text { EasySep }^{\mathrm{TM}} \\
\text { (StemCell } \\
\text { Technologies) }\end{array}$ & $\begin{array}{l}\text { Pre-negative magnetic CD45 } \\
\text { enrichment to exclude hematogenous } \\
\text { cells; secondary enrichment with } \\
\text { EpCAM and CK; manual CTC } \\
\text { enumeration }\end{array}$ & $\begin{array}{l}\text { Little clinical relevance of } \\
\text { CTC number to CRC } \\
\text { staging; CTC morphology } \\
\text { and phenotype closely } \\
\text { related to CRC stages }\end{array}$ & $\begin{array}{l}72 \%(41 / 57 \\
\text { patients) }\end{array}$ & Case control & $\begin{array}{c}\text { CRC } \\
\text { screening }\end{array}$ & [102] \\
\hline & CD45 & $\begin{array}{l}\text { CK18, CEP8, } \\
\text { DAPI }\end{array}$ & $\begin{array}{l}\text { SE-iFISH } \\
\text { (Cytelligen) }\end{array}$ & $\begin{array}{l}\text { Pre-negative CD45 enrichment to } \\
\text { exclude hematogenous cells; secondary } \\
\text { enrichment with EpCAM and CK with } \\
\text { anti-CK18 and anti-CEP8 }\end{array}$ & $\begin{array}{c}\text { Identification of CTC } \\
\text { optimal detection time } \\
\text { (after at least } 7 \\
\text { postoperative days) } \\
\end{array}$ & $\begin{array}{c}85 \%(17 / 20 \\
\text { patients })\end{array}$ & $\begin{array}{l}\text { Cross-sectional } \\
\text { cohort study }\end{array}$ & $\begin{array}{c}\text { CRC } \\
\text { screening and } \\
\text { postoperative } \\
\text { monitoring }\end{array}$ & [101] \\
\hline $\begin{array}{l}\text { Combined } \\
\text { approach }\end{array}$ & CD45 & $\begin{array}{c}\text { CK3 } \\
\text { CK3, CK19, } \\
\text { MUC1, CD44, } \\
\text { CD133, ALDH1, }\end{array}$ & $\begin{array}{l}\text { CellSearch }{ }^{\circledR} \text { system } \\
\text { (Veridex) } \\
\text { CellSearch + } \\
\text { cytomorphology } \\
\text { + FACS + } \\
\text { RT-qPCR }\end{array}$ & $\begin{array}{l}\text { Negative enrichment; CTCs were } \\
\text { isolated via CD45+ cells depletion kit } \\
\text { and further enriched with } \\
\text { anti-CK3-labeled magnetic beads. } \\
\text { Combination of several CTC-negative } \\
\text { enrichment techniques }\end{array}$ & $\begin{array}{c}\text { CTCs as novel therapeutic } \\
\text { targets for nonmetastatic } \\
\text { CRC } \\
\text { Improved sensitivity and } \\
\text { specificity }\end{array}$ & $\begin{array}{c}54 \% \\
(34 / 63 \text { patients }) \\
68.3 \%(34 / 43 \\
\text { patients })\end{array}$ & Comparative study & $\begin{array}{c}\text { CRC } \\
\text { screening }\end{array}$ & [98] \\
\hline
\end{tabular}

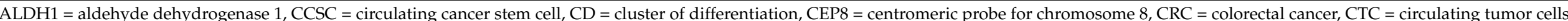

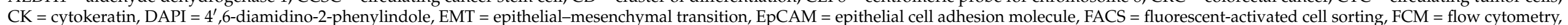

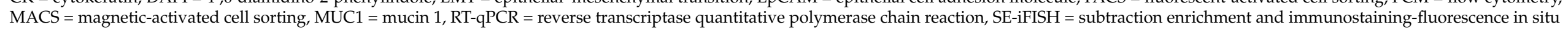
hybridization, VIM = vimentin. 


\section{Importance of Enrichment Technique over Selection of CTC Analysis and Characterization in the CRC Screening Stage}

Ideally, CTC enrichment would provide pure CTC for enumeration, as well as downstream analysis, and reflect the total CTC status in CRC patients. As one of the commonly used techniques, biophysical property-based enrichment systems are capable of isolating CTCs via the specific selection of size, density, or deformability. Although a wider subset of CTCs could be enriched due to its independence on the CSM, the recovery efficiency is limited due to the buildup of filtration resistance, formation of CTC aggregates, and membrane clogging [125,126].

On the other hand, functional and nucleic acid-based CTC enrichment are widely used alternatives to immunoaffinity-based systems. These techniques identify specific tumor markers to confirm the presence of CTCs indirectly [127,128]. For instance, the immunocytochemistry of CTCs permits CTC morphological analysis and labeling of specific ligands $[129,130]$, whereas qRT-PCR allows the detection of specific biomarkers with high sensitivity [131]. Post-functional assays, where enriched CTCs are cultured in 2D or 3D models, also enable the evaluation of migration and invasion abilities [132,133]. The advancement in NGS technologies even granted the possibility of dissecting CTC at the single-cell level [134]. Nonetheless, they lack specificity due to the potential to capture noncancerous cells to generate false-positive signals, thus decreasing the overall accuracy. The lack of standardized sampling and pre-enrichment methods might also result in the significant loss of CTCs. Other fatal clinical implications include (1) high contamination risks with hematopoietic cells/white blood cells; (2) denaturation/lysis of CTCs; (3) lack of certain CTC subpopulations due to unspecific markers during enrichment; and (4) the isolated CTCs might not reflect the actual CTC status of patients, resulting in bias or false results. In our opinion, these limitations could be overcome if functional and nucleic acid-based CTC enrichment techniques are incorporated as downstream analysis for CTC analysis and characterization. Thus, we believe that the multi-CSM-based CTC isolation system is a promising enrichment strategy due to its higher CTC capture efficiency and higher specificity. Moreover, the enumeration of enriched CTCs, alone, has been proven to be adequate for CRC screening, prognosis, and disease progressing monitoring [135]. Table 2 summarizes advantages and disadvantages of single and multi-CSM based enrichment over other CTC enrichment methods.

Table 2. Comparisons of most commonly used CTC enrichment methods.

\begin{tabular}{|c|c|c|}
\hline CTC Enrichment Technique & Advantages & Disadvantages \\
\hline $\begin{array}{c}\text { Biophysical isolation } \\
\text { (size/microfiltration; density gradient } \\
\text { centrifugation) }\end{array}$ & $\begin{array}{l}\text { Quick and simple way to isolate CTCs; } \\
\text { Label-free CTC isolation; } \\
\text { Rapid processing of large volumes; } \\
\text { Applicable to all types of cancers; } \\
\text { Inexpensive; } \\
\text { Harvest a wider subsets of CTCs }\end{array}$ & $\begin{array}{l}\text { Poor sensitivity due to the loss of some } \\
\text { CTC during migration or formation of } \\
\text { CTC aggregates or membrane clogging; } \\
\text { Low specificity; } \\
\text { Stringent sampling procedure (blood } \\
\text { samples collected must be processed } \\
\text { immediately and required } \\
\text { pre-enrichment step); } \\
\text { High contamination risks with } \\
\text { hematopoietic cells; } \\
\text { Limited due to the heterogeneity in the } \\
\text { size and density of CTCs }\end{array}$ \\
\hline
\end{tabular}


Table 2. Cont.

CTC Enrichment Technique

Single CSM-based system

(a) Positive enrichment

(b) Negative enrichment

\section{Advantages}

Clinically validated (FDA-approved

system);

Robust and reproducible;

Specific to certain CTC subpopulation

depending on the selected marker

(epithelial or mesenchymal trait);

Advancement in microfluidics technology

allows intact cells for downstream

analysis

Capable of harvesting all types of CTCs if

negative enrichment was applied;

High CTC viability;

No bias based on CSMs;

More competent for the discovery of

cellular and transcriptomic cancer

biomarkers of cancer and downstream

analyses such as genetic assays, CTC

culture, and xenografts
Multi-CSM-based system

Higher yield than single CSM-based
enrichment systems;
High CTC capture efficiency (CTCs of
different origins were captured by
covering epithelial, mesenchymal, and
stem cell markers);
Increased analytic sensitivity and
specificity than single CSM-based system;
Capable of addressing the totality of
CTCs;
Advancement in microfluidics technology
allows intact cells for downstream
analysis

Higher yield than single CSM-based enrichment systems

High CTC capture efficiency (CTCs of different origins were captured by covering epithelial, mesenchymal, and Capable of addressing the totality of CTCs; allows intact cells for downstream analysis

CCSC-targeted CTC enrichment

\section{Lack of specific combinatorial list of} CSMs;

Unstandardized protocols when multiple markers are used (e.g., marker concentrations and incubation time); Lack of automated procedures;

Limited studies using multiple CSMs for pre-CTC enrichment step

$$
\begin{aligned}
& \text { Identification of CTCs with cancer stem } \\
& \text { cell characteristics; } \\
& \text { More selection of CSMs (including Lrg5, } \\
& \text { DCLK1, and ANXA2); } \\
& \text { More specific for mCRC screening; } \\
& \text { Drug resistance identification }
\end{aligned}
$$

Nucleic acid-based or functional-based
enrichment system/post-CTC analysis
and characterization (e.g.,
immunocytochemistry, qRT-PCR, ddPCR,
EPISPOT, NGS, and functional assays)

Detection of viable CTCs;

Evaluation of CTC migration and invasion abilities;

Ability to address cellular heterogeneity;

Capable of CTC characterization;

Capable of single cell resolution analysis;

Permit CTC morphology analysis;

Detection of specific markers not limited

to the surface of CTCs

\section{Low population in CTCs; \\ Limited studies}

Significant loss of certain CTC

subpopulations if a single CSM is used

positive enrichment

(e.g., EMT and mutations) due to the use of single CSM

Inability to address totality of CTCs;

Low purity and specificity due to the loss enrichment;

Uncertainty in the accuracy to identify a patient's CTC status 
become the main focus for CTC enrichment. However, there is an ongoing debate between CSM and circulating tumor markers in the blood, where the latter could potentially detect CTCs without the isolation from whole blood [136]. The concept of a circulating tumor marker applies to a chemical product originated from a CRC cell (including CTC), such that its concentration in the blood represents a quantifiable assessment of the tumor burden at a specific time [137].

Some examples for the blood-based circulating tumor marker include proteins [46,138-140]; low-molecular-weight metabolites (volatile organic compounds) [141]; DNA (including methylation markers) [142-147]; and RNA (messenger RNA, non-coding RNA, and microRNA) [148-151]. These blood biomarkers are also capable of the detection of tumorspecific mutations associated with the response to targeted therapy. Nevertheless, the analytical specificity might be limited as they could potentially be released from necrotic or apoptotic cells, as well as active secretions by intact cells, of tumor origin or/and from nontumor origins, including hematopoietic, immune, and blood stromal cells [152-155].

In 2020, Liu et al. discovered three identical mutations in both cell-free DNA (cfDNA) and CTCs, seven mutations found only in the cfDNA, and one exon 19 deletion in the CTCs from 11 EGFR-mutated cancer patients. Their results proved the supremacy of the combination of cfDNA and CTCs over either test alone. Interestingly, in the third subsequent blood draw, the previous exon 19 deletion could not be detected. The reduction in CTC concentration due to chemotherapy/cell apoptosis resulted in DNA from CTCs being released into the blood, resulting in the better performance of cfDNA than CTCs. In other words, new mutations would be first detected in the CTCs, while cfDNA would provide a snapshot of dying cancer cells instead [156]. Consequently, instead of replacing CSM-dependent CTC enrichment, blood-based circulating tumor markers represent complementary predictive cancer biomarkers, as well as real-time CRC monitoring in clinical practice [157].

\section{Challenges in Routine Implementation of CTC-Specific CSM-dependent CRC Detection}

Despite the discovery of numerous CTC-specific CSMs, the main limitation that hampers existing CTC detection technologies is still the a priori knowledge of the exact protein composition on the CTCs surfaces, and the lack of a universal marker(s) to address the heterogeneity of CTCs in CRC $[125,158,159]$. The current gold-standard technique for CTC detection, the microscopic cell imaging, also presents many drawbacks such as the low number of markers, inability to analyze multiple markers simultaneously in routine use, long turnaround time (incompatible with the urgent need for delivery of treatment), and the requirements for specific laboratory instruments and professional expertise (pathologists) for data analysis [160,161]. Furthermore, the lack of large-population follow-up cohort studies increases the difficulties of translating current CSM-based CTC detection methods into the clinical setting for CRC screening, diagnosis, prognosis, real-time monitoring, and therapeutic response [50-54]. Other reasons include (i) the vast number of methods described for potential CTC detection (including the pre-analytical, analytical, and postanalytical phases), without a consensus on the ideal/standardized technical approach; (ii) difficulty in controlling the pre-analytical phase to obtain robust and reproducible results; and (iii) the high cost of the currently available techniques [162,163].

\section{Conclusions}

Cell surface markers/antigens on CTCs are crucial markers for the diagnosis and prognosis of metastatic and nonmetastatic CRC. Despite the potential scientific and medical usefulness of current CTC enrichment technologies, adopting them into the clinical setting will demand laborious studies into their analytical validity, clinical validity, and clinical utility. Therefore, the standardization of all procedures should be emphasized. A multi-marker-based system is believed to permit the enrichment of a wider subset of CTCs, including phenotypes of epithelial, mesenchymal, and those transitioned in between. Nevertheless, additional large-scale studies in high-risk groups and the further under- 
standing of their biology and significance could enhance CTCs' utility as a blood-based biomarker [163]. Finally, a real gap exists between the genuine attraction of obtaining a large number of publications in this domain and its application into routine clinical practice.

Author Contributions: Conceptualization, N.-S.A.M. and F.Y.F.T.; writing-original draft preparation, F.Y.F.T. and N.-S.A.M.; preparation of figures, F.Y.F.T.; provide critical scientific insights, N.-S.A.M., N.A., S.N.N. and L.-H.L. All authors have read and agreed to the published version of the manuscript.

Funding: This review is supported by Higher Institution Centre of Excellence fund (JJ-2021-002) by Ministry of Higher Education, Dana Impak Perdana Grant (DIP-2018-010) by Universiti Kebangsaan Malaysia (UKM) (Kuala Lumpur, Malaysia) and Monash University Malaysia (Selangor, Malaysia).

Acknowledgments: The authors thank Universiti Kebangsaan Malaysia (UKM) (Kuala Lumpur, Malaysia) for awarding the research grant, and Monash University Malaysia (Selangor, Malaysia) for supporting the article processing charges.

Conflicts of Interest: The authors declare no conflict of interest. The authors declare that the research was conducted in the absence of any commercial or financial relationships that could be construed as a potential conflict of interest. The funders had no role in the design of the study; in the collection, analyses, or interpretation of data; in the writing of the manuscript, or in the decision to publish the results.

\section{Abbreviations}

$\begin{array}{ll}\text { AJCC } & \text { American Joint Committee on Cancer } \\ \text { CCSCCD } & \text { Circulating cancer stem cellsCluster of differentiation } \\ \text { cfDNA } & \text { Cell-free DNA } \\ \text { CK } & \text { Cytokeratin } \\ \text { CRC } & \text { Colorectal cancer } \\ \text { CSM } & \text { Cell surface marker } \\ \text { CTC } & \text { Circulating tumor cell } \\ \text { DAPI } & 4^{\prime} \text {,6-diamidino-2-phenylindole } \\ \text { EMT } & \text { Epithelial-mesenchymal transition } \\ \text { EpCAM } & \text { Epithelial cell adhesion molecule } \\ \text { FACS } & \text { Fluorescent-activated cell sorting } \\ \text { FCM } & \text { Flow cytometry } \\ \text { FDA } & \text { Food and Drug Administration } \\ \text { KRAS } & \text { Kirsten rat sarcoma viral oncogene } \\ \text { MACS } & \text { Magnetic-activated cell sorting } \\ \text { mCRC } & \text { Metastatic CRC } \\ \text { pan-CK } & \text { Pan-cytokeratin } \\ \text { RT-qPCR } & \text { Reverse-transcriptase quantitative polymerase chain reaction } \\ \text { SE-iFISH } & \text { Subtraction enrichment and immunostaining-fluorescence in situ hybridization } \\ \text { VIM } & \text { vimentin }\end{array}$

\section{References}

1. Bray, F.; Ferlay, J.; Soerjomataram, I.; Siegel, R.L.; Torre, L.A.; Jemal, A. Global cancer statistics 2018: GLOBOCAN estimates of incidence and mortality worldwide for 36 cancers in 185 countries. CA Cancer J. Clin. 2018, 68, 394-424. [CrossRef] [PubMed]

2. Siegel, R.L.; Miller, K.D.; Jemal, A. Cancer statistics, 2019: Cancer Statistics, 2019. CA Cancer J. Clin. 2019, 69, 7-34. [CrossRef] [PubMed]

3. Van der Jeught, K.; Xu, H.-C.; Li, Y.-J.; Lu, X.-B.; Ji, G. Drug resistance and new therapies in colorectal cancer. World J. Gastroenterol. 2018, 24, 3834-3848. [CrossRef] [PubMed]

4. Arvelo, F. Biology of colorectal cancer. Ecancermedicalscience 2015, 9, 520. [CrossRef]

5. Engstrand, J.; Nilsson, H.; Strömberg, C.; Jonas, E.; Freedman, J. Colorectal cancer liver metastases-A population-based study on incidence, management and survival. BMC Cancer 2018, 18, 78. [CrossRef]

6. Chakraborty, S.; Rahman, T. The difficulties in cancer treatment. Ecancermedicalscience 2012, 6, ed16. [CrossRef]

7. Hazewinkel, Y.; Dekker, E. Colonoscopy: Basic principles and novel techniques. Nat. Rev. Gastroenterol. Hepatol. 2011, 8, 554-564. [CrossRef] 
8. Makaju, R.; Amatya, M.; Sharma, S.; Dhakal, R.; Bhandari, S.; Shrestha, S.; Gurung, R.; Malla, B. Clinico-Pathological Correlation of Colorectal Diseases by Colonoscopy and Biopsy. Kathmandu Univ. Med. J. 2017, 58, 173-178.

9. Carroll, M.R.R.; Seaman, H.E.; Halloran, S.P. Tests and investigations for colorectal cancer screening. Clin. Biochem. 2014, 47, 921-939. [CrossRef]

10. Pox, C.P.; Altenhofen, L.; Brenner, H.; Theilmeier, A.; Stillfried, D.V.; Schmiegel, W. Efficacy of a Nationwide Screening Colonoscopy Program for Colorectal Cancer. Gastroenterology 2012, 142, 1460-1467.e2. [CrossRef]

11. Marzouk, O.; Schofield, J. Review of Histopathological and Molecular Prognostic Features in Colorectal Cancer. Cancers 2011, 3 , 2767-2810. [CrossRef]

12. Amin, M.B.; Greene, F.L.; Edge, S.B.; Compton, C.C.; Gershenwald, J.E.; Brookland, R.K.; Meyer, L.; Gress, D.M.; Byrd, D.R.; Winchester, D.P. The Eighth Edition AJCC Cancer Staging Manual: Continuing to build a bridge from a population-based to a more "personalized" approach to cancer staging. CA Cancer J. Clin. 2017, 67, 93-99. [CrossRef]

13. Kim, A. Imaging Diagnosis of Colorectal Cancer. J. Korean Med. Assoc. 2010, 53, 562-568. [CrossRef]

14. Brenner, H.; Hoffmeister, M.; Arndt, V.; Stegmaier, C.; Altenhofen, L.; Haug, U. Protection from Right- and Left-Sided Colorectal Neoplasms After Colonoscopy: Population-Based Study. J. Natl Cancer Inst. 2010, 102, 89-95. [CrossRef]

15. Lansdorp-Vogelaar, I.; Knudsen, A.B.; Brenner, H. Cost-effectiveness of colorectal cancer screening. Epidemiol. Rev. 2011, 33, 88-100. [CrossRef]

16. Punt, C.J.A.; Koopman, M.; Vermeulen, L. From tumour heterogeneity to advances in precision treatment of colorectal cancer. Nat. Rev. Clin. Oncol. 2017, 14, 235-246. [CrossRef]

17. Wolf, A.M.D.; Fontham, E.T.H.; Church, T.R.; Flowers, C.R.; Guerra, C.E.; LaMonte, S.J.; Etzioni, R.; McKenna, M.T.; Oeffinger, K.C.; Shih, Y.-C.T.; et al. Colorectal cancer screening for average-risk adults: 2018 guideline update from the American Cancer Society. CA Cancer J. Clin. 2018, 68, 250-281. [CrossRef]

18. Zhai, Z.; Yu, X.; Yang, B.; Zhang, Y.; Zhang, L.; Li, X.; Sun, H. Colorectal cancer heterogeneity and targeted therapy: Clinical implications, challenges and solutions for treatment resistance. Semin. Cell Dev. Biol. 2017, 64, 107-115. [CrossRef]

19. Shaukat, A.; Mongin, S.J.; Geisser, M.S.; Lederle, F.A.; Bond, J.H.; Mandel, J.S.; Church, T.R. Long-term mortality after screening for colorectal cancer. N. Engl. J. Med. 2013, 369, 1106-1114. [CrossRef]

20. Batth, I.S.; Mitra, A.; Manier, S.; Ghobrial, I.M.; Menter, D.; Kopetz, S.; Li, S. Circulating tumor markers: Harmonizing the yin and yang of CTCs and ctDNA for precision medicine. Ann. Oncol. 2017, 28, 468-477. [CrossRef]

21. Marcuello, M.; Vymetalkova, V.; Neves, R.P.L.; Duran-Sanchon, S.; Vedeld, H.M.; Tham, E.; van Dalum, G.; Flügen, G.; GarciaBarberan, V.; Fijneman, R.J.; et al. Circulating biomarkers for early detection and clinical management of colorectal cancer. Mol. Asp. Med. 2019, 69, 107-122. [CrossRef]

22. Micalizzi, D.S.; Maheswaran, S.; Haber, D.A. A conduit to metastasis: Circulating tumor cell biology. Genes Dev. 2017, 31, 1827-1840. [CrossRef]

23. Dive, C.; Brady, G. SnapShot: Circulating Tumor Cells. Cell 2017, 168, 742-742.e1. [CrossRef]

24. Hardingham, J.E.; Grover, P.; Winter, M.; Hewett, P.J.; Price, T.J.; Thierry, B. Detection and Clinical Significance of Circulating Tumor Cells in Colorectal Cancer-20 Years of Progress. Mol. Med. 2015, 21, S25-S31. [CrossRef]

25. Hardingham, J.E.; Hewett, P.J.; Sage, R.E.; Finch, J.L.; Nuttall, J.D.; Kotasek, D.; Dobrovic, A. Molecular detection of blood-borne epithelial cells in colorectal cancer patients and in patients with benign bowel disease. Int. J. Cancer 2000, 89, 8-13. [CrossRef]

26. Pantel, K.; Speicher, M.R. The biology of circulating tumor cells. Oncogene 2016, 35, 1216-1224. [CrossRef]

27. Pantel, K.; Denève, E.; Nocca, D.; Coffy, A.; Vendrell, J.-P.; Maudelonde, T.; Riethdorf, S.; Alix-Panabières, C. Circulating epithelial cells in patients with benign colon diseases. Clin. Chem. 2012, 58, 936-940. [CrossRef]

28. Souza, E.; Silva, V.; Abdallah, E.A.; de Mello, C.A.L.; Tariki, M.S.; Calsavara, V.F.; Chinen, L.T.D. Prospective study with circulating tumor cells as potential prognosis biomarker in metastatic colorectal cancer. JCO 2020, 38, 203. [CrossRef]

29. Freeman, J.; Gray, E.S.; Ziman, M. Circulating Tumor Cells as Biomarkers in Cancer. In Biomarkers in Cancer. Biomarkers in Disease: Methods, Discoveries and Applications; Preedy, V., Patel, V., Eds.; Springer: Dordrecht, The Netherlands, 2015; pp. 31-51. [CrossRef]

30. Tsai, W.-S.; You, J.-F.; Hung, H.-Y.; Hsieh, P.-S.; Hsieh, B.; Lenz, H.-J.; Idos, G.; Friedland, S.; Yi-Jiun Pan, J.; Shao, H.-J.; et al. Novel Circulating Tumor Cell Assay for Detection of Colorectal Adenomas and Cancer. Clin. Transl. Gastroenterol. 2019, 10, e00088. [CrossRef]

31. Chang, Y.S.; di Tomaso, E.; McDonald, D.M.; Jones, R.; Jain, R.K.; Munn, L.L. Mosaic blood vessels in tumors: Frequency of cancer cells in contact with flowing blood. Proc. Natl. Acad. Sci. USA 2000, 97, 14608-14613. [CrossRef]

32. Tamimi, Y. Micrometastatic Circulating Tumor cells; A Challenge for an Early Detection and Better Survival Rates. J. Carcinog. Mutagenesis 2015, 6, 9.

33. Oakman, C.; Pestrin, M.; Bessi, S.; Galardi, F.; Di Leo, A. Significance of Micrometastases: Circulating Tumor Cells and Disseminated Tumor Cells in Early Breast Cancer. Cancers 2010, 2, 1221-1235. [CrossRef] [PubMed]

34. Koyanagi, K.; Bilchik, A.J.; Saha, S.; Turner, R.R.; Wiese, D.; McCarter, M.; Shen, P.; Deacon, L.; Elashoff, D.; Hoon, D.S.B. Prognostic Relevance of Occult Nodal Micrometastases and Circulating Tumor Cells in Colorectal Cancer in a Prospective Multicenter Trial. Clin. Cancer Res. 2008, 14, 7391-7396. [CrossRef] [PubMed]

35. Molnar, B.; Floro, L.; Sipos, F.; Toth, B.; Sreter, L.; Tulassay, Z. Elevation in Peripheral Blood Circulating Tumor Cell Number Correlates with Macroscopic Progression in UICC Stage IV Colorectal Cancer Patients. Available online: https://www.hindawi. com/journals/dm/2008/941509/ (accessed on 1 October 2020). 
36. De Wit, S.; van Dalum, G.; Terstappen, L.W.M.M. Detection of Circulating Tumor Cells. Available online: https:/ /www.hindawi. com/journals/scientifica/2014/819362/ (accessed on 1 October 2020).

37. Ferreira, M.M.; Ramani, V.C.; Jeffrey, S.S. Circulating tumor cell technologies. Mol. Oncol. 2016, 10, 374-394. [CrossRef]

38. Thery, L.; Meddis, A.; Cabel, L.; Proudhon, C.; Latouche, A.; Pierga, J.-Y.; Bidard, F.-C. Circulating Tumor Cells in Early Breast Cancer. JNCI Cancer Spectr. 2019, 3, pkz026. [CrossRef]

39. Allard, W.J.; Terstappen, L.W.M.M. CCR 20th Anniversary Commentary: Paving the Way for Circulating Tumor Cells. Clin. Cancer Res. 2015, 21, 2883-2885. [CrossRef]

40. Tieng, F.Y.F.; Baharudin, R.; Abu, N.; Mohd Yunos, R.-I.; Lee, L.-H.; Ab Mutalib, N.-S. Single Cell Transcriptome in Colorectal Cancer-Current Updates on Its Application in Metastasis, Chemoresistance and the Roles of Circulating Tumor Cells. Front. Pharmacol. 2020, 11, 135. [CrossRef]

41. Tieng, F.Y.F.; Abu, N.; Lee, L.-H.; Ab Mutalib, N.-S. Microsatellite Instability in Colorectal Cancer Liquid Biopsy-Current Updates on Its Potential in Non-Invasive Detection, Prognosis and as a Predictive Marker. Diagnostics 2021, 11, 544. [CrossRef]

42. Berretta, M.; Alessandrini, L.; De Divitiis, C.; Nasti, G.; Lleshi, A.; Di Francia, R.; Facchini, G.; Cavaliere, C.; Buonerba, C.; Canzonieri, V. Serum and tissue markers in colorectal cancer: State of art. Crit. Rev. Oncol. Hematol. 2017, 111, 103-116. [CrossRef]

43. Bhardwaj, M.; Gies, A.; Werner, S.; Schrotz-King, P.; Brenner, H. Blood-Based Protein Signatures for Early Detection of Colorectal Cancer: A Systematic Review. Clin. Transl. Gastroenterol. 2017, 8, e128. [CrossRef]

44. Huang, Z.; Huang, D.; Ni, S.; Peng, Z.; Sheng, W.; Du, X. Plasma microRNAs are promising novel biomarkers for early detection of colorectal cancer. Int. J. Cancer 2010, 127, 118-126. [CrossRef]

45. Liu, Z.; Zhang, Y.; Niu, Y.; Li, K.; Liu, X.; Chen, H.; Gao, C. A Systematic Review and Meta-Analysis of Diagnostic and Prognostic Serum Biomarkers of Colorectal Cancer. PLoS ONE 2014, 9, e103910. [CrossRef]

46. Tieng, F.Y.F.; Abu, N.; Sukor, S.; Mohd Azman, Z.A.; Mahamad Nadzir, N.; Lee, L.-H.; Ab Mutalib, N.S. L1CAM, CA9, KLK6, HPN, and ALDH1A1 as Potential Serum Markers in Primary and Metastatic Colorectal Cancer Screening. Diagnostics 2020, 10, 444. [CrossRef]

47. Torres, A.; Pac-Sosińska, M.; Wiktor, K.; Paszkowski, T.; Maciejewski, R.; Torres, K. CD44, TGM2 and EpCAM as novel plasma markers in endometrial cancer diagnosis. BMC Cancer 2019, 19, 401. [CrossRef]

48. Vatandoost, N.; Ghanbari, J.; Mojaver, M.; Avan, A.; Ghayour-Mobarhan, M.; Nedaeinia, R.; Salehi, R. Early detection of colorectal cancer: From conventional methods to novel biomarkers. J. Cancer Res. Clin. Oncol. 2016, 142, 341-351. [CrossRef]

49. Hon, K.W.; Abu, N.; Ab Mutalib, N.-S.; Jamal, R. Exosomes as Potential Biomarkers and Targeted Therapy in Colorectal Cancer: A Mini-Review. Front. Pharmacol. 2017, 8, 583. [CrossRef]

50. Huang, M.-Y.; Tsai, H.-L.; Huang, J.-J.; Wang, J.-Y. Clinical Implications and Future Perspectives of Circulating Tumor Cells and Biomarkers in Clinical Outcomes of Colorectal Cancer. Transl. Oncol. 2016, 9, 340-347. [CrossRef]

51. Kanaan, Z.; Roberts, H.; Eichenberger, M.; Billeter, A.; Ocheretner, G.; Pan, J.; Rai, S.; Jorden, J.; Williford, A.; Galandiuk, S. A Plasma MicroRNA Panel for Detection of Colorectal Adenomas: A Step Toward More Precise Screening for Colorectal Cancer. Ann. Surg. 2013, 258, 400-408. [CrossRef]

52. Bünger, S.; Haug, U.; Kelly, F.M.; Klempt-Giessing, K.; Cartwright, A.; Posorski, N.; Dibbelt, L.; Fitzgerald, S.P.; Bruch, H.-P.; Roblick, U.J.; et al. Toward standardized high-throughput serum diagnostics: Multiplex-protein array identifies IL-8 and VEGF as serum markers for colon cancer. J. Biomol. Screen 2011, 16, 1018-1026. [CrossRef]

53. Vocka, M.; Langer, D.; Fryba, V.; Petrtyl, J.; Hanus, T.; Kalousova, M.; Zima, T.; Petruzelka, L. Novel serum markers HSP60, CHI3L1, and IGFBP-2 in metastatic colorectal cancer. Oncol. Lett. 2019, 18, 6284-6292. [CrossRef]

54. Imperiale, T.F.; Imperiale, T.F. Noninvasive Screening Tests for Colorectal Cancer. DDI 2012, 30, 16-26. [CrossRef]

55. Hanke, B.; Riedel, C.; Lampert, S.; Happich, K.; Martus, P.; Parsch, H.; Himmler, B.; Hohenberger, W.; Hahn, E.G.; Wein, A. CEA and CA19-9 measurement as a monitoring parameter in metastatic colorectal cancer (CRC) under palliative first-line chemotherapy with weekly 24-hour infusion of high-dose 5-fluorouracil (5-FU) and folinic acid (FA). Ann. Oncol. 2001, 12, 221-226. [CrossRef]

56. Hauptman, N.; Glavač, D. Colorectal Cancer Blood-Based Biomarkers. Gastroenterol. Res. Pr. 2017, 2017. [CrossRef]

57. Negm, R.S.; Verma, M.; Srivastava, S. The promise of biomarkers in cancer screening and detection. Trends Mol. Med. 2002, 8, 288-293. [CrossRef]

58. Rupert, K.; Holubec, L.; Nosek, J.; Houdek, K.; Topolcan, O.; Treska, V. Significance of the TPS cytokeratin marker in the postoperative follow up of colorectal carcinoma patients. Rozhl. Chir. 2009, 88, 428-433. [PubMed]

59. Świderska, M.; Choromańska, B.; Dabrowska, E.; Konarzewska-Duchnowska, E.; Choromańska, K.; Szczurko, G.; Myśliwiec, P.; Dadan, J.; Ładny, J.R.; Zwierz, K. The diagnostics of colorectal cancer. Contemp. Oncol. 2014, 18, 1-6. [CrossRef]

60. Locker, G.Y.; Hamilton, S.; Harris, J.; Jessup, J.M.; Kemeny, N.; Macdonald, J.S.; Somerfield, M.R.; Hayes, D.F.; Bast, R.C.; ASCO. ASCO 2006 update of recommendations for the use of tumor markers in gastrointestinal cancer. J. Clin. Oncol. 2006, 24, 5313-5327. [CrossRef] [PubMed]

61. Guadagni, F.; Roselli, M.; Cosimelli, M.; Mannella, E.; Tedesco, M.; Cavaliere, F.; Grassi, A.; Abbolito, M.R.; Greiner, J.W.; Schlom, J. TAG-72 (CA 72-4 assay) as a complementary serum tumor antigen to carcinoembryonic antigen in monitoring patients with colorectal cancer. Cancer 1993, 72, 2098-2106. [CrossRef]

62. Jelski, W.; Mroczko, B. Biochemical Markers of Colorectal Cancer-Present and Future. Cancer Manag. Res. 2020, 12, $4789-4797$. [CrossRef] 
63. Quentmeier, A.; Möller, P.; Schwarz, V.; Abel, U.; Schlag, P. Carcinoembryonic antigen, CA 19-9, and CA 125 in normal and carcinomatous human colorectal tissue. Cancer 1987, 60, 2261-2266. [CrossRef]

64. Ryan, E.J.; Creagh, E.M. Emerging methods in colorectal cancer screening. Br. J. Surg. 2018, 105, e16-e18. [CrossRef]

65. Tian, C.; Xu, X.; Wang, Y.; Li, D.; Lu, H.; Yang, Z. Development and Clinical Prospects of Techniques to Separate Circulating Tumor Cells from Peripheral Blood. Cancer Manag. Res. 2020, 12, 7263-7275. [CrossRef]

66. Hayes, D.F.; Smerage, J.B. Circulating Tumor Cells. In Progress in Molecular Biology and Translational Science; Elsevier: Amsterdam, The Netherlands, 2010; Volume 95, pp. 95-112. [CrossRef]

67. Krebs, M.G.; Hou, J.-M.; Ward, T.H.; Blackhall, F.H.; Dive, C. Circulating tumour cells: Their utility in cancer management and predicting outcomes. Ther. Adv. Med. Oncol. 2010, 2, 351-365. [CrossRef]

68. Miller, M.C.; Doyle, G.V.; Terstappen, L.W.M.M. Significance of Circulating Tumor Cells Detected by the CellSearch System in Patients with Metastatic Breast Colorectal and Prostate Cancer. J. Oncol. 2010, 2010, 617421. [CrossRef]

69. Andree, K.C.; van Dalum, G.; Terstappen, L.W.M.M. Challenges in circulating tumor cell detection by the CellSearch system. Mol. Oncol. 2016, 10, 395-407. [CrossRef]

70. Van der Toom, E.E.; Verdone, J.E.; Gorin, M.A.; Pienta, K.J. Technical challenges in the isolation and analysis of circulating tumor cells. Oncotarget 2016, 7, 62754-62766. [CrossRef]

71. Zou, D.; Cui, D. Advances in isolation and detection of circulating tumor cells based on microfluidics. Cancer Biol. Med. 2018, 15, 335-353. [CrossRef]

72. Vafaei, S.; Roudi, R.; Madjd, Z.; Aref, A.R.; Ebrahimi, M. Potential theranostics of circulating tumor cells and tumor-derived exosomes application in colorectal cancer. Cancer Cell Int. 2020, 20, 288. [CrossRef]

73. Bankó, P.; Lee, S.Y.; Nagygyörgy, V.; Zrínyi, M.; Chae, C.H.; Cho, D.H.; Telekes, A. Technologies for circulating tumor cell separation from whole blood. J. Hematol. Oncol. 2019, 12, 48. [CrossRef]

74. Rothé, F.; Maetens, M.; Rouas, G.; Paesmans, M.; Van den Eynde, M.; Van Laethem, J.-L.; Vergauwe, P.; Deboever, G.; Bareche, Y.; Vandeputte, C.; et al. CTCs as a prognostic and predictive biomarker for stage II/III Colon Cancer: A companion study to the PePiTA trial. BMC Cancer 2019, 19, 304. [CrossRef]

75. Cristofanilli, M.; Budd, G.T.; Ellis, M.J.; Stopeck, A.; Matera, J.; Miller, M.C.; Reuben, J.M.; Doyle, G.V.; Allard, W.J.; Terstappen, L.W.M.M.; et al. Circulating tumor cells, disease progression, and survival in metastatic breast cancer. N. Engl. J. Med. 2004, 351, 781-791. [CrossRef]

76. Millner, L.M.; Linder, M.W.; Valdes, R. Circulating Tumor Cells: A Review of Present Methods and the Need to Identify Heterogeneous Phenotypes. Ann. Clin. Lab. Sci. 2013, 43, 295-304. [PubMed]

77. Cohen, S.J.; Punt, C.J.A.; Iannotti, N.; Saidman, B.H.; Sabbath, K.D.; Gabrail, N.Y.; Picus, J.; Morse, M.A.; Mitchell, E.; Miller, M.C.; et al. Prognostic significance of circulating tumor cells in patients with metastatic colorectal cancer. Ann. Oncol. 2009, 20, 1223-1229. [CrossRef]

78. Cohen, S.J.; Punt, C.J.; lannotti, N.; Saidman, B.H.; Sabbath, K.D.; Gabrail, N.Y.; Picus, J.; Morse, M.; Mitchell, E.; Miller, M.C.; et al. Relationship of Circulating Tumor Cells to Tumor Response, Progression-Free Survival, and Overall Survival in Patients with Metastatic Colorectal Cancer. J. Clin. Oncol. 2008, 26, 3213-3221. [CrossRef]

79. Mamdouhi, T.; Twomey, J.D.; McSweeney, K.M.; Zhang, B. Fugitives on the run: Circulating tumor cells (CTCs) in metastatic diseases. Cancer Metastasis Rev. 2019, 38, 297-305. [CrossRef]

80. Kowalik, A.; Kowalewska, M.; Góźdź, S. Current approaches for avoiding the limitations of circulating tumor cells detection methods-implications for diagnosis and treatment of patients with solid tumors. Transl. Res. 2017, 185, 58-84.e15. [CrossRef]

81. Lin, Z.; Luo, G.; Du, W.; Kong, T.; Liu, C.; Liu, Z. Recent Advances in Microfluidic Platforms Applied in Cancer Metastasis: Circulating Tumor Cells' (CTCs) Isolation and Tumor-On-A-Chip. Small 2020, 16, 1903899. [CrossRef]

82. Gupta, P.; Gulzar, Z.; Hsieh, B.; Lim, A.; Watson, D.; Mei, R. Analytical validation of the CellMax platform for early detection of cancer by enumeration of rare circulating tumor cells. J. Circ. Biomark. 2019, 8, 1849454419899214. [CrossRef]

83. Kure, K.; Hosoya, M.; Ueyama, T.; Fukaya, M.; Sugimoto, K.; Tomiki, Y.; Ohnaga, T.; Sakamoto, K.; Komiyama, H. Using the polymeric circulating tumor cell chip to capture circulating tumor cells in blood samples of patients with colorectal cancer. Oncol. Lett 2020, 19, 2286-2294. [CrossRef]

84. Toh, J.W.T.; Lim, S.H.; MacKenzie, S.; de Souza, P.; Bokey, L.; Chapuis, P.; Spring, K.J. Association between Microsatellite Instability Status and Peri-Operative Release of Circulating Tumour Cells in Colorectal Cancer. Cells 2020, 9, 425. [CrossRef]

85. Mármol, I.; Sánchez-de-Diego, C.; Pradilla Dieste, A.; Cerrada, E.; Rodriguez Yoldi, M.J. Colorectal Carcinoma: A General Overview and Future Perspectives in Colorectal Cancer. Int. J. Mol. Sci. 2017, 18, 197. [CrossRef] [PubMed]

86. Huels, D.J.; Sansom, O.J. Stem vs non-stem cell origin of colorectal cancer. Br. J. Cancer 2015, 113, 1-5. [CrossRef] [PubMed]

87. Eslami-S, Z.; Cortés-Hernández, L.E.; Alix-Panabières, C. Epithelial Cell Adhesion Molecule: An Anchor to Isolate Clinically Relevant Circulating Tumor Cells. Cells 2020, 9, 1836. [CrossRef] [PubMed]

88. Alix-Panabières, C.; Mader, S.; Pantel, K. Epithelial-mesenchymal plasticity in circulating tumor cells. J. Mol. Med. 2017, 95, 133-142. [CrossRef] [PubMed]

89. Fischer, K.R.; Durrans, A.; Lee, S.; Sheng, J.; Li, F.; Wong, S.T.C.; Choi, H.; El Rayes, T.; Ryu, S.; Troeger, J.; et al. Epithelialto-mesenchymal transition is not required for lung metastasis but contributes to chemoresistance. Nature 2015, 527, $472-476$. [CrossRef] 
90. Yu, M.; Bardia, A.; Wittner, B.S.; Stott, S.L.; Smas, M.E.; Ting, D.T.; Isakoff, S.J.; Ciciliano, J.C.; Wells, M.N.; Shah, A.M.; et al. Circulating breast tumor cells exhibit dynamic changes in epithelial and mesenchymal composition. Science 2013, 339, 580-584. [CrossRef]

91. Zheng, X.; Carstens, J.L.; Kim, J.; Scheible, M.; Kaye, J.; Sugimoto, H.; Wu, C.-C.; LeBleu, V.S.; Kalluri, R. Epithelial-to-mesenchymal transition is dispensable for metastasis but induces chemoresistance in pancreatic cancer. Nature 2015, 527, 525-530. [CrossRef]

92. Kalluri, R.; Weinberg, R.A. The basics of epithelial-mesenchymal transition. J. Clin. Investig. 2009, 119, 1420-1428. [CrossRef]

93. Dongre, A.; Weinberg, R.A. New insights into the mechanisms of epithelial-mesenchymal transition and implications for cancer. Nat. Rev. Mol. Cell Biol. 2019, 20, 69-84. [CrossRef]

94. Barrière, G.; Tartary, M.; Rigaud, M. Epithelial Mesenchymal Transition: A New Insight into the Detection of Circulating Tumor Cells. Available online: https://www.hindawi.com/journals/isrn/2012/382010/ (accessed on 21 February 2021).

95. Zhao, R.; Cai, Z.; Li, S.; Cheng, Y.; Gao, H.; Liu, F.; Wu, S.; Liu, S.; Dong, Y.; Zheng, L.; et al. Expression and clinical relevance of epithelial and mesenchymal markers in circulating tumor cells from colorectal cancer. Oncotarget 2017, 8, 9293-9302. [CrossRef]

96. Dizdar, L.; Fluegen, G.; van Dalum, G.; Honisch, E.; Neves, R.P.; Niederacher, D.; Neubauer, H.; Fehm, T.; Rehders, A.; Krieg, A.; et al. Detection of circulating tumor cells in colorectal cancer patients using the GILUPI CellCollector: Results from a prospective, single-center study. Mol. Oncol. 2019, 13, 1548-1558. [CrossRef]

97. Kang, H.; Kim, J.; Cho, H.; Han, K.-H. Evaluation of Positive and Negative Methods for Isolation of Circulating Tumor Cells by Lateral Magnetophoresis. Micromachines 2019, 10, 386. [CrossRef]

98. Bahnassy, A.A.; Salem, S.E.; Mohanad, M.; Abulezz, N.Z.; Abdellateif, M.S.; Hussein, M.; Zekri, C.A.N.; Zekri, A.-R.N.; Allahloubi, N.M.A. Prognostic significance of circulating tumor cells (CTCs) in Egyptian non-metastatic colorectal cancer patients: A comparative study for four different techniques of detection (Flowcytometry, CellSearch, Quantitative Real-time PCR and Cytomorphology). Exp. Mol. Pathol. 2019, 106, 90-101. [CrossRef]

99. Wang, L.; Zhou, S.; Zhang, W.; Wang, J.; Wang, M.; Hu, X.; Liu, F.; Zhang, Y.; Jiang, B.; Yuan, H. Circulating tumor cells as an independent prognostic factor in advanced colorectal cancer: A retrospective study in 121 patients. Int. J. Colorectal Dis. 2019, 34, 589-597. [CrossRef]

100. Soler, A.; Cayrefourcq, L.; Mazel, M.; Alix-Panabières, C. EpCAM-Independent Enrichment and Detection of Viable Circulating Tumor Cells Using the EPISPOT Assay. In Circulating Tumor Cells: Methods and Protocols; Magbanua, M.J.M., Park, J.W., Eds.; Methods in Molecular Biology; Springer: New York, NY, USA, 2017; Volume 1634, pp. 263-276. [CrossRef]

101. Huang, T.; Xu, C.; Xiao, J.; Wang, Q.; Wang, Y.; Zhang, Y.; Bai, D.; Zhou, F.; Zhao, X. Determination of the optimal detection time of circulating tumor cells for the postoperative monitoring of colorectal cancer. Oncol. Lett. 2020, 19, 2996-3002. [CrossRef]

102. Hamid, F.-B.; Islam, F.; Lu, C.-T.; Matos, M.; Cheng, T.; Gopalan, V.; Lam, A.K. Abstract 5367: Identification and clinical value of the circulating tumor cells (CTCs) in the colorectal cancer. Cancer Res. 2020, 80, 5367. [CrossRef]

103. Bahrami, A.; Hassanian, S.M.; ShahidSales, S.; Farjami, Z.; Hasanzadeh, M.; Anvari, K.; Aledavood, A.; Maftouh, M.; Ferns, G.A.; Khazaei, M.; et al. Targeting RAS signaling pathway as a potential therapeutic target in the treatment of colorectal cancer. J. Cell. Physiol. 2018, 233, 2058-2066. [CrossRef]

104. Neuzillet, C.; Tijeras-Raballand, A.; de Mestier, L.; Cros, J.; Faivre, S.; Raymond, E. MEK in cancer and cancer therapy. Pharmacol. Ther. 2014, 141, 160-171. [CrossRef]

105. Prieur, A.; Cappellini, M.; Habif, G.; Lefranc, M.-P.; Mazard, T.; Morency, E.; Pascussi, J.-M.; Flacelière, M.; Cahuzac, N.; Vire, B.; et al. Targeting the Wnt Pathway and Cancer Stem Cells with Anti-progastrin Humanized Antibodies as a Potential Treatment for K-RAS-Mutated Colorectal Cancer. Clin. Cancer Res. 2017, 23, 5267-5280. [CrossRef]

106. Sullivan, K.M.; Kozuch, P.S. Impact of KRAS Mutations on Management of Colorectal Carcinoma. Available online: https: //www.hindawi.com/journals/pri/2011/219309/ (accessed on 21 February 2021).

107. Amado, R.G.; Wolf, M.; Peeters, M.; Van Cutsem, E.; Siena, S.; Freeman, D.J.; Juan, T.; Sikorski, R.; Suggs, S.; Radinsky, R.; et al. Wild-Type KRAS Is Required for Panitumumab Efficacy in Patients with Metastatic Colorectal Cancer. JCO 2008, 26, $1626-1634$. [CrossRef]

108. Allegra, C.J.; Jessup, J.M.; Somerfield, M.R.; Hamilton, S.R.; Hammond, E.H.; Hayes, D.F.; McAllister, P.K.; Morton, R.F.; Schilsky, R.L. American Society of Clinical Oncology Provisional Clinical Opinion: Testing for KRAS Gene Mutations in Patients with Metastatic Colorectal Carcinoma to Predict Response to Anti-Epidermal Growth Factor Receptor Monoclonal Antibody Therapy. JCO 2009, 27, 2091-2096. [CrossRef]

109. De Roock, W.; Claes, B.; Bernasconi, D.; De Schutter, J.; Biesmans, B.; Fountzilas, G.; Kalogeras, K.T.; Kotoula, V.; Papamichael, D.; Laurent-Puig, P.; et al. Effects of KRAS, BRAF, NRAS, and PIK3CA mutations on the efficacy of cetuximab plus chemotherapy in chemotherapy-refractory metastatic colorectal cancer: A retrospective consortium analysis. Lancet Oncol. 2010, 11, 753-762. [CrossRef]

110. Van Cutsem, E.; Lenz, H.-J.; Köhne, C.-H.; Heinemann, V.; Tejpar, S.; Melezínek, I.; Beier, F.; Stroh, C.; Rougier, P.; van Krieken, J.H.; et al. Fluorouracil, Leucovorin, and Irinotecan Plus Cetuximab Treatment and RAS Mutations in Colorectal Cancer. JCO 2015, 33, 692-700. [CrossRef]

111. Feng, C.; Wang, J.; Yang, X.; Zang, X.; Zhou, H.; Zhang, E.; Li, H.; Liu, B.; Chen, S.; Wang, Y.; et al. Construction and Characterization of KRAS Immune Lipid Magnetic Balls for Colorectal Cancer Circulating Tumor Cells. Cancer Manag. Res. 2020, 12, 10067-10075. [CrossRef] 
112. Lopresti, A.; Malergue, F.; Bertucci, F.; Liberatoscioli, M.L.; Garnier, S.; DaCosta, Q.; Finetti, P.; Gilabert, M.; Raoul, J.L.; Birnbaum, D.; et al. Sensitive and easy screening for circulating tumor cells by flow cytometry. JCI Insight 2019, 4, e128180. [CrossRef]

113. Wilson, R.E.; O'Connor, R.; Gallops, C.E.; Kwizera, E.A.; Noroozi, B.; Morshed, B.I.; Wang, Y.; Huang, X. Immunomagnetic Capture and Multiplexed Surface Marker Detection of Circulating Tumor Cells with Magnetic Multicolor Surface-Enhanced Raman Scattering Nanotags. ACS Appl. Mater. Interfaces 2020, 12, 47220-47232. [CrossRef]

114. Agnoletto, C.; Corrà, F.; Minotti, L.; Baldassari, F.; Crudele, F.; Cook, W.J.J.; Di Leva, G.; d'Adamo, A.P.; Gasparini, P.; Volinia, S. Heterogeneity in Circulating Tumor Cells: The Relevance of the Stem-Cell Subset. Cancers 2019, 11, 483. [CrossRef]

115. Grillet, F.; Bayet, E.; Villeronce, O.; Zappia, L.; Lagerqvist, E.L.; Lunke, S.; Charafe-Jauffret, E.; Pham, K.; Molck, C.; Rolland, N.; et al. Circulating tumour cells from patients with colorectal cancer have cancer stem cell hallmarks in ex vivo culture. Gut 2017, 66, 1802-1810. [CrossRef]

116. Barbazán, J.; Alonso-Alconada, L.; Muinelo-Romay, L.; Vieito, M.; Abalo, A.; Alonso-Nocelo, M.; Candamio, S.; Gallardo, E.; Fernández, B.; Abdulkader, I.; et al. Molecular Characterization of Circulating Tumor Cells in Human Metastatic Colorectal Cancer. PLoS ONE 2012, 7, e40476. [CrossRef]

117. Garg, M. Epithelial, mesenchymal and hybrid epithelial/mesenchymal phenotypes and their clinical relevance in cancer metastasis. Expert Rev. Mol. Med. 2017, 19, e3. [CrossRef]

118. Polyak, K.; Weinberg, R.A. Transitions between epithelial and mesenchymal states: Acquisition of malignant and stem cell traits. Nat. Rev. Cancer 2009, 9, 265-273. [CrossRef] [PubMed]

119. Yang, M.-H.; Imrali, A.; Heeschen, C. Circulating cancer stem cells: The importance to select. Chin. J. Cancer Res. 2015, 27, 437-449. [CrossRef] [PubMed]

120. Kantara, C.; O'Connell, M.; Luthra, G.; Gajjar, A.; Sarkar, S.; Ullrich, R.; Singh, P. Methods for Detecting Circulating Cancer Stem Cells (CCSCs) as a Novel Approach for Diagnosis of Colon Cancer Relapse/Metastasis. Lab. Investig. 2015, 95, 100-112. [CrossRef] [PubMed]

121. Todaro, M.; Gaggianesi, M.; Catalano, V.; Benfante, A.; Iovino, F.; Biffoni, M.; Apuzzo, T.; Sperduti, I.; Volpe, S.; Cocorullo, G.; et al. CD44v6 Is a Marker of Constitutive and Reprogrammed Cancer Stem Cells Driving Colon Cancer Metastasis. Cell Stem Cell 2014, 14, 342-356. [CrossRef]

122. Zhou, Y.; Xia, L.; Wang, H.; Oyang, L.; Su, M.; Liu, Q.; Lin, J.; Tan, S.; Tian, Y.; Liao, Q.; et al. Cancer stem cells in progression of colorectal cancer. Oncotarget 2018, 9, 33403-33415. [CrossRef]

123. Ma, L.; Dong, L.; Chang, P. CD44v6 engages in colorectal cancer progression. Cell Death Dis. 2019, 10, 30. [CrossRef]

124. Nicolazzo, C.; Loreni, F.; Caponnetto, S.; Magri, V.; Vestri, A.R.; Zamarchi, R.; Gradilone, A.; Facchinetti, A.; Rossi, E.; Cortesi, E.; et al. Baseline CD44v6-positive circulating tumor cells to predict first-line treatment failure in patients with metastatic colorectal cancer. Oncotarget 2020, 11, 4115-4122. [CrossRef]

125. Habli, Z.; AlChamaa, W.; Saab, R.; Kadara, H.; Khraiche, M.L. Circulating Tumor Cell Detection Technologies and Clinical Utility: Challenges and Opportunities. Cancers 2020, 12, 1930. [CrossRef]

126. Gabriel, M.T.; Calleja, L.R.; Chalopin, A.; Ory, B.; Heymann, D. Circulating Tumor Cells: A Review of Non-EpCAM-Based Approaches for Cell Enrichment and Isolation. Clin. Chem. 2016, 62, 571-581. [CrossRef]

127. Cayrefourcq, L.; Alix-Panabières, C. CTCs as Liquid Biopsy: Where Are We Now? IntechOpen: London, UK, 2019. [CrossRef]

128. Esmaeilsabzali, H.; Beischlag, T.V.; Cox, M.E.; Parameswaran, A.M.; Park, E.J. Detection and isolation of circulating tumor cells: Principles and methods. Biotechnol. Adv. 2013, 31, 1063-1084. [CrossRef]

129. Kang, Y.-T.; Kim, Y.J.; Lee, T.; Cho, Y.-H.; Chang, H.J.; Lee, H.-M. Cytopathological Study of the Circulating Tumor Cells filtered from the Cancer Patients' Blood using Hydrogel-based Cell Block Formation. Sci. Rep. 2018, 8, 15218. [CrossRef]

130. Fehm, T.; Solomayer, E.; Meng, S.; Tucker, T.; Lane, N.; Wang, J.; Gebauer, G. Methods for isolating circulating epithelial cells and criteria for their classification as carcinoma cells. Cytotherapy 2005, 7, 171-185. [CrossRef]

131. Yadegarazari, R.; Hassanzadeh, T.; Majlesi, A.; Keshvari, A.; Monsef Esfahani, A.; Tootoonchi, A.; Shabab, N.; Saidijam, M. Improved Real-Time RT-PCR Assays of Two Colorectal Cancer Peripheral Blood mRNA Biomarkers: A Pilot Study. Iran. Biomed. J. 2013, 17, 15-21. [CrossRef]

132. Akpe, V.; Kim, T.H.; Brown, C.L.; Cock, I.E. Circulating tumour cells: A broad perspective. J. R. Soc. Interface 2020, 17, 20200065. [CrossRef]

133. Pallante, P.; Pisapia, P.; Bellevicine, C.; Malapelle, U.; Troncone, G. Circulating Tumour Cells in Predictive Molecular Pathology: Focus on Drug-Sensitive Assays and 3D Culture. ACY 2019, 63, 171-181. [CrossRef]

134. Cheng, Y.-H.; Chen, Y.-C.; Lin, E.; Brien, R.; Jung, S.; Chen, Y.-T.; Lee, W.; Hao, Z.; Sahoo, S.; Min Kang, H.; et al. Hydro-Seq enables contamination-free high-throughput single-cell RNA-sequencing for circulating tumor cells. Nat. Commun. 2019, 10, 2163. [CrossRef]

135. Tsai, W.-S.; Chen, J.-S.; Shao, H.-J.; Wu, J.-C.; Lai, J.-M.; Lu, S.-H.; Hung, T.-F.; Chiu, Y.-C.; You, J.-F.; Hsieh, P.-S.; et al. Circulating Tumor Cell Count Correlates with Colorectal Neoplasm Progression and Is a Prognostic Marker for Distant Metastasis in Non-Metastatic Patients. Sci. Rep. 2016, 6, 24517. [CrossRef]

136. Vafaei, S.; Fattahi, F.; Ebrahimi, M.; Janani, L.; Shariftabrizi, A.; Madjd, Z. Common molecular markers between circulating tumor cells and blood exosomes in colorectal cancer: A systematic and analytical review. CMAR 2019, 11, 8669-8698. [CrossRef] 
137. Horwich, A.; Ross, G. Circulating Tumor Markers. In Principles of Molecular Oncology; Bronchud, M.H., Foote, M.A., Peters, W.P., Robinson, M.O., Eds.; Humana Press: Totowa, NJ, USA, 2000; pp. 111-124. [CrossRef]

138. Kuppusamy, P.; Govindan, N.; Yusoff, M.M.; Ichwan, S.J.A. Proteins are potent biomarkers to detect colon cancer progression. Saudi J. Biol. Sci. 2017, 24, 1212-1221. [CrossRef]

139. Ahn, S.B.; Sharma, S.; Mohamedali, A.; Mahboob, S.; Redmond, W.J.; Pascovici, D.; Wu, J.X.; Zaw, T.; Adhikari, S.; Vaibhav, V.; et al. Potential early clinical stage colorectal cancer diagnosis using a proteomics blood test panel. Clin. Proteom. 2019, 16, 34. [CrossRef]

140. Borrebaeck, C.A.K. Precision diagnostics: Moving towards protein biomarker signatures of clinical utility in cancer. Nat. Rev. Cancer 2017, 17, 199-204. [CrossRef]

141. Loktionov, A. Biomarkers for detecting colorectal cancer non-invasively: DNA, RNA or proteins? World J. Gastrointest. Oncol. 2020, 12, 124-148. [CrossRef]

142. Berger, B.M.; Ahlquist, D.A. Stool DNA screening for colorectal neoplasia: Biological and technical basis for high detection rates. Pathology 2012, 44, 80-88. [CrossRef]

143. Liu, R.; Su, X.; Long, Y.; Zhou, D.; Zhang, X.; Ye, Z.; Ma, J.; Tang, T.; Wang, F.; He, C. A systematic review and quantitative assessment of methylation biomarkers in fecal DNA and colorectal cancer and its precursor, colorectal adenoma. Mutat. Res. 2019, 779, 45-57. [CrossRef]

144. Worm Ørntoft, M.-B. Review of Blood-Based Colorectal Cancer Screening: How Far Are Circulating Cell-Free DNA Methylation Markers from Clinical Implementation? Clin. Colorectal Cancer 2018, 17, e415-e433. [CrossRef]

145. Rasmussen, S.L.; Krarup, H.B.; Sunesen, K.G.; Johansen, M.B.; Stender, M.T.; Pedersen, I.S.; Madsen, P.H.; Thorlacius-Ussing, O. Hypermethylated DNA, a circulating biomarker for colorectal cancer detection. PLoS ONE 2017, 12, e0180809. [CrossRef]

146. Ab Mutalib, N.-S.; Md Yusof, N.F.; Abdul, S.-N.; Jamal, R. Pharmacogenomics DNA Biomarkers in Colorectal Cancer: Current Update. Front. Pharmacol. 2017, 8, 736. [CrossRef]

147. Ab Mutalib, N.-S.; Baharuddin, R.; Jamal, R. Epigenome-Wide Analysis of DNA Methylation in Colorectal Cancer. In Computational Epigenetics and Diseases; Elsevier: Amsterdam, The Netherlands, 2019; pp. 289-310. [CrossRef]

148. Luo, X.; Burwinkel, B.; Tao, S.; Brenner, H. MicroRNA Signatures: Novel Biomarker for Colorectal Cancer? Cancer Epidemiol. Biomark. Prev. 2011, 20, 1272-1286. [CrossRef] [PubMed]

149. Abedini, P.; Fattahi, A.; Agah, S.; Talebi, A.; Beygi, A.H.; Amini, S.M.; Mirzaei, A.; Akbari, A. Expression analysis of circulating plasma long noncoding RNAs in colorectal cancer: The relevance of lncRNAs ATB and CCAT1 as potential clinical hallmarks. J. Cell. Physiol. 2019, 234, 22028-22033. [CrossRef] [PubMed]

150. Chen, B.; Xia, Z.; Deng, Y.-N.; Yang, Y.; Zhang, P.; Zhu, H.; Xu, N.; Liang, S. Emerging microRNA biomarkers for colorectal cancer diagnosis and prognosis. Open Biol. 2019, 9, 180212. [CrossRef] [PubMed]

151. Bastaminejad, S.; Taherikalani, M.; Ghanbari, R.; Akbari, A.; Shabab, N.; Saidijam, M. Investigation of MicroRNA-21 Expression Levels in Serum and Stool as a Potential Non-Invasive Biomarker for Diagnosis of Colorectal Cancer. Iran. Biomed. J. 2017, 21, 106-113. [CrossRef] [PubMed]

152. Merker, J.D.; Oxnard, G.R.; Compton, C.; Diehn, M.; Hurley, P.; Lazar, A.J.; Lindeman, N.; Lockwood, C.M.; Rai, A.J.; Schilsky, R.L.; et al. Circulating Tumor DNA Analysis in Patients with Cancer: American Society of Clinical Oncology and College of American Pathologists Joint Review. J. Clin. Oncol. 2018, 36, 1631-1641. [CrossRef] [PubMed]

153. Stewart, C.M.; Kothari, P.D.; Mouliere, F.; Mair, R.; Somnay, S.; Benayed, R.; Zehir, A.; Weigelt, B.; Dawson, S.-J.; Arcila, M.E.; et al. The value of cell-free DNA for molecular pathology. J. Pathol. 2018, 244, 616-627. [CrossRef] [PubMed]

154. Fiala, C.; Diamandis, E.P. New approaches for detecting cancer with circulating cell-free DNA. BMC Med. 2019, 17, 159. [CrossRef] [PubMed]

155. Alix-Panabières, C.; Pantel, K. Clinical Applications of Circulating Tumor Cells and Circulating Tumor DNA as Liquid Biopsy. Cancer Discov. 2016, 6, 479-491. [CrossRef] [PubMed]

156. Liu, H.E.; Vuppalapaty, M.; Wilkerson, C.; Renier, C.; Chiu, M.; Lemaire, C.; Che, J.; Matsumoto, M.; Carroll, J.; Crouse, S.; et al. Detection of EGFR Mutations in cfDNA and CTCs, and Comparison to Tumor Tissue in Non-Small-Cell-Lung-Cancer (NSCLC) Patients. Front. Oncol. 2020, 10, 572895. [CrossRef]

157. Ilie, M.; Hofman, V.; Long, E.; Bordone, O.; Selva, E.; Washetine, K.; Marquette, C.H.; Hofman, P. Current challenges for detection of circulating tumor cells and cell-free circulating nucleic acids, and their characterization in non-small cell lung carcinoma patients. What is the best blood substrate for personalized medicine? Ann. Transl. Med. 2014, 2, 107. [CrossRef]

158. Agashe, R.; Kurzrock, R. Circulating Tumor Cells: From the Laboratory to the Cancer Clinic. Cancers 2020, 12, 2361. [CrossRef]

159. Yang, C.; Xia, B.-R.; Jin, W.-L.; Lou, G. Circulating tumor cells in precision oncology: Clinical applications in liquid biopsy and 3D organoid model. Cancer Cell Int. 2019, 19, 341. [CrossRef]

160. Ciurte, A.; Selicean, C.; Soritau, O.; Buiga, R. Automatic detection of circulating tumor cells in darkfield microscopic images of unstained blood using boosting techniques. PLoS ONE 2018, 13, e0208385. [CrossRef]

161. Salvianti, F.; Gelmini, S.; Costanza, F.; Mancini, I.; Sonnati, G.; Simi, L.; Pazzagli, M.; Pinzani, P. The pre-analytical phase of the liquid biopsy. N. Biotechnol. 2020, 55, 19-29. [CrossRef] 
162. Dickinson, B.T.; Kisiel, J.; Ahlquist, D.A.; Grady, W.M. Molecular markers for colorectal cancer screening. Gut 2015, 64, 1485-1494. [CrossRef]

163. Ijzerman, M.J.; de Boer, J.; Azad, A.; Degeling, K.; Geoghegan, J.; Hewitt, C.; Hollande, F.; Lee, B.; To, Y.H.; Tothill, R.W.; et al. Towards Routine Implementation of Liquid Biopsies in Cancer Management: It Is Always Too Early, until Suddenly It Is Too Late. Diagnostics 2021, 11, 103. [CrossRef] 